A two-dimensional analytical model and experimental validation of garter stitch knitted shape memory alloy actuator architecture

This article has been downloaded from IOPscience. Please scroll down to see the full text article. 2012 Smart Mater. Struct. 21085011

(http://iopscience.iop.org/0964-1726/21/8/085011)

View the table of contents for this issue, or go to the journal homepage for more

Download details:

IP Address: 141.211.173.82

The article was downloaded on 26/06/2013 at 14:47

Please note that terms and conditions apply. 


\title{
A two-dimensional analytical model and experimental validation of garter stitch knitted shape memory alloy actuator architecture
}

\author{
Julianna Abel, Jonathan Luntz and Diann Brei \\ Department of Mechanical Engineering, University of Michigan, 2250 G G Brown, 2350 Hayward \\ Street, Ann Arbor, MI 48109, USA \\ E-mail: jmariee@umich.edu (J Abel)
}

Received 26 July 2011, in final form 17 January 2012

Published 20 July 2012

Online at stacks.iop.org/SMS/21/085011

\begin{abstract}
Active knits are a unique architectural approach to meeting emerging smart structure needs for distributed high strain actuation with simultaneous force generation. This paper presents an analytical state-based model for predicting the actuation response of a shape memory alloy (SMA) garter knit textile. Garter knits generate significant contraction against moderate to large loads when heated, due to the continuous interlocked network of loops of SMA wire. For this knit architecture, the states of operation are defined on the basis of the thermal and mechanical loading of the textile, the resulting phase change of the SMA, and the load path followed to that state. Transitions between these operational states induce either stick or slip frictional forces depending upon the state and path, which affect the actuation response. A load-extension model of the textile is derived for each operational state using elastica theory and Euler-Bernoulli beam bending for the large deformations within a loop of wire based on the stress-strain behavior of the SMA material. This provides kinematic and kinetic relations which scale to form analytical transcendental expressions for the net actuation motion against an external load. This model was validated experimentally for an SMA garter knit textile over a range of applied forces with good correlation for both the load-extension behavior in each state as well as the net motion produced during the actuation cycle $(250 \%$ recoverable strain and over $50 \%$ actuation). The two-dimensional analytical model of the garter stitch active knit provides the ability to predict the kinetic actuation performance, providing the basis for the design and synthesis of large stroke, large force distributed actuators that employ this novel architecture.
\end{abstract}

(Some figures may appear in colour only in the online journal)

\section{Nomenclature}

$\mathbf{A}$
$\mathbf{B}$
$\mathbf{C}$
$C$
$C_{1}, C_{2}$,
$C_{3}, C_{4}$
$\mathbf{D}$

A

B

$C_{3}, C_{4}$
Inflection point at end of loop leg

Interlacing contact point

Point at top of loop

Course height

Functions of angles used to simplify notation

Center of the unit cell
D

$d$

E

$E_{\mathrm{A}}$

$E_{\mathrm{M}}$

$\hat{E}$

F

$F_{\text {app }}$
Knitting needle diameter

Wire diameter

Elliptic integral of the second kind

Base material austenite Young's modulus

Base material martensite Young's modulus

Difference between complete and incomplete elliptic integrals of the second kind Elliptic integral of the first kind Externally applied force to knit textile 


$\begin{array}{ll}F_{\text {loop }} & \text { Externally applied force to knit loop } \\ F_{\mathrm{UC}} & \text { Externally applied force to unit cell } \\ \hat{F} & \begin{array}{l}\text { Difference between complete and incomplete } \\ \text { elliptic integrals of the first kind }\end{array} \\ I & \text { Second moment of inertia of wire cross-section } \\ k_{1}, k_{2} & \text { Geometric functions of angles used in elliptic } \\ & \text { integrals } \\ L & \text { Knit loop length } \\ L_{\mathrm{subscript}} & \text { State dependent length of knit textile } \\ M & \text { Bending moment at top of loop } \\ N_{\mathrm{c}} & \text { Number of courses in knit textile } \\ N_{\mathrm{W}} & \text { Number of wales in knit textile } \\ O & \text { Unit cell origin } \\ P & \text { Reaction force at end of loop leg } \\ R & \text { Reaction force at interlacing contact point } \\ s & \text { Length along loop } \\ T & \text { Horizontal wire tension at top of loop } \\ W & \text { Wale width } \\ X, Y & \text { Global coordinate system } \\ x, y & \text { Local coordinate system for segment } \mathbf{B C} \\ x^{\prime}, y^{\prime} & \text { Local coordinate system for segment AB } \\ \alpha & \text { Loop state angle of the connecting leg at } \mathbf{A} \\ \beta & \text { Loop state angle of the reaction force between } \\ \Delta & \text { adjacent loops } \\ \delta & \text { Knit textile deflection } \\ \varepsilon_{1}, \varepsilon_{2}, & \text { Unit cell deflection } \\ \varphi_{1 \mathrm{~B}}, \varphi_{2 B} & \text { Geometric functions of angles used in elliptic } \\ \mu & \text { integrals } \\ \theta, \theta^{\prime} & \text { Loop state angle of force } P \text { at } \mathbf{A} \\ & \text { Coefficient of friction between interlacing loops } \\ \text { Deflection angle along loop }\end{array}$

Subscripts

$\begin{array}{ll}\text { A } & \text { Austenite phase } \\ \text { ACT } & \text { Actuator } \\ \text { cont } & \text { Contracted state } \\ \text { ext } & \text { Extended state } \\ \text { M } & \text { Martensite phase } \\ \text { w } & \text { Initial state with zero external load }\end{array}$

\section{Introduction}

Across diverse fields such as transportation, medicine, and communications, recent research has indicated high promise if distributed, large-amplitude contractile actuation were available. For example, in transportation, contractile actuation such as active skins [1] and morphing control surfaces [2, 3] can significantly reduce drag, increasing fuel economy and enabling diverse aerodynamic performance envelopes [4]. To achieve these goals, it is necessary to provide large strains under aerodynamic loads, which is a challenge for current actuation technology. Similar needs arise in medical devices [5, 6]. One application, a catheter actuated by off-axis contractile shape memory alloy (SMA) wire, has made improved navigation through the body possible and has reduced stress to surrounding tissue during deployment of arthroscopic devices [7]. While SMA actuated catheters of current research interest are able to overcome the modest structural forces of the catheter sheath, they cannot provide the desired level of dexterity to fully navigate bodily conduits because they are limited by the maximum contractile strains of the SMA material. Communication infrastructure, such as antennas, space telescopes and solar arrays, have a continual demand for large deployment sizes with precise control of shape despite delivery vehicle constraints and high launch costs [8-12]. Because of these actuation demands, communication devices can also benefit from the development of distributed large contractile surface actuation in structures. Large strain contractile actuators that could provide the required surface strains to reassemble large, complex systems in space would reduce the size and complexity of deployment mechanisms. These diverse applications illustrate a common need for distributed large strain contractile actuation under usable forces that is difficult to meet with current actuation technology.

While research has advanced actuator capabilities, providing large distributed actuation strains against application forces is still difficult with traditional and smart material actuators. Conventional technologies such as electromagnetics, hydraulics, and pneumatics come close to producing the necessary kinetic behavior but they have many drawbacks including large size and weight, substantial infrastructures (such as pumps and generators), and lack of mechanical and environmental robustness in addition to actuator specific problems such as strong electromagnetic signatures and hydraulic leakage. Smart material based actuators have long held the promise of increasing the actuation authority and decreasing weight/size by an order of magnitude because of their high energy/power densities [13] (up to two or three orders of magnitude for shape memory alloys versus electromagnetic actuators). Smart materials can also generate distributed actuation which conventional actuators cannot directly accomplish. Unfortunately, smart materials by themselves either have very low strokes resulting from material strains of only a fraction of a percent (piezoceramics, electrostrictives, magnetostrictives) [14-17] or low forces, typically on the order of milliNewtons up to at most one Newton (piezopolymers, electroactive polymers, etc) [18-23]. To overcome these material shortcomings, substantial research has been conducted on strain amplification architectures over the last decade [24-40], these strategies normally result in point actuators with relatively small strains. To move toward distributed actuation, active composite architectures using piezoelectrics [41-53] and shape memory alloys [54-60] have been developed. While these smart material architectures have improved actuator performance, large strains (over 50\%) with any reasonable force generation (tens to hundreds of Newtons) have remained elusive.

Through the development of advanced smart material actuation architectures, distributed large contractile actuation motions can be created. Active knits, a novel cellular textile architecture containing a periodic continuous interlocked network of loops of active material, produce large distributed actuation profiles that could enhance actuation capabilities. All knitted textiles (traditional and active) are differentiated from other textile architectures (weaving, braiding, stitching, etc) by their unique unit cells-the knit and purl loops 


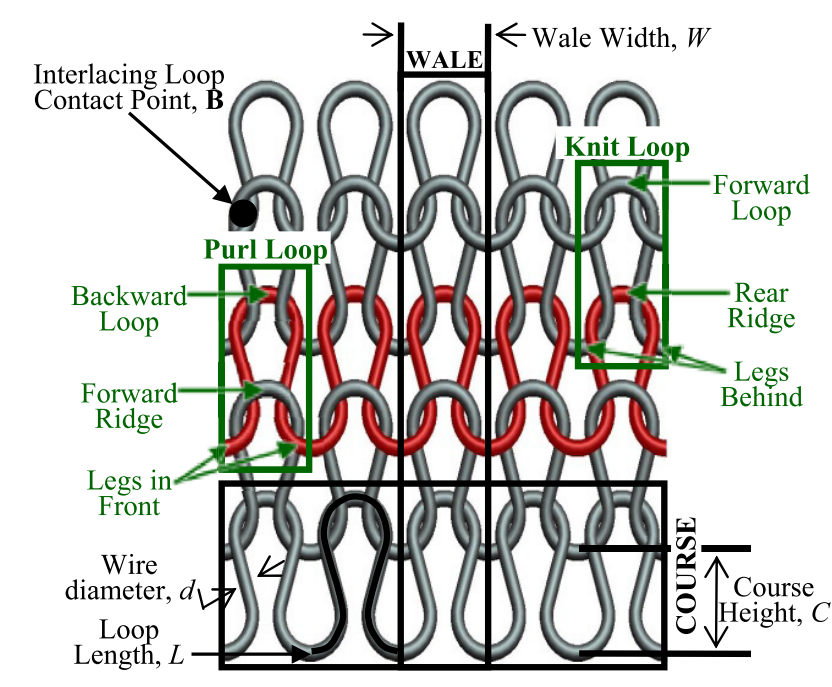

Figure 1. Basic garter stitch architecture. Forward knit loops and backward purl loops make up courses (rows of loops) and wales (columns of loops), characterized by the course height $(C)$, wale width $(W)$, wire diameter $(d)$, and loop length $(L)$.

(figure 1). These unit cells are composed of an $180^{\circ}$ bulbshaped loop extending into two legs that interlock with a ridge formed by the previous row. The loops are distinguished by the position of the ridge (rear ridge for knit loop, forward ridge for purl loop) and can be arranged to provide a wide variety of stitches. The most common stitch, garter stitch, has a symmetric arrangement of alternating rows of knit and purl loops (figure 1). This symmetric architecture allows active garter stitch knitted actuators to supply the most basic actuation motion-planar contraction. Upon actuation the smart material fiber tries to return to its original, stress-free straight shape by recovering the strain induced in the smart material fiber during loop formation. The fiber straightening causes the height of the individual loops to decrease (while slightly expanding the width) and the loops to slip relative to each other in the plane in which they lie. The change in loop geometry and the interaction between rows of adjacent loops combine to reduce the total length of the active knit textile which results in textiles with net strains that are orders of magnitude higher than the local strains within the material itself. While any contractile smart material could be used in the garter stitch knitted architecture, Dynalloy's Flexinol ${ }^{\circledR}$ shape memory alloy wire is used to investigate this architectural paradigm, because it was inexpensive, robust, and readily available. Garter stitch active knit actuators knitted with Flexinol ${ }^{\circledR}$ wire have demonstrated large strains in excess of $50 \%$ and forces in the tens of Newtons [61], but larger strains, up to $100 \%$ or more, with hundreds of Newtons of force are possible through manipulation of geometric parameters and scaling of the textile, enabling applications across diverse fields.

The garter stitch active knit architecture is a promising actuation architecture, however, no predictive models for active knit actuators which can capture the complex operational and material transitions between states within the actuation cycle currently exist. Various models of passive knitted textiles exist and these can provide a starting point for modeling active knit textiles. Purely geometric, small deformation models of plain knit apparel fabrics have been developed over the last century [62-74]. More recently, models of knitted engineering materials (such as glass, steel, and carbon fiber) have been derived to predict the performance of engineering composites, which may improve mechanical performance (energy absorption, bearing and notched strengths, and fracture toughness) [75-79]. While more sophisticated models have been developed [75-82], they still apply only to traditional passive engineering materials, modeling only a single tensile operational state and only a single phase of the material. None of these models account for the changes in material phase of an active material, the thermal and tensile loading states experienced during an actuation cycle, or the internal friction forces and kinematic constraints induced by the transitions between these states, and therefore, they are not easily adaptable for modeling actuation.

This paper presents an analytical state-based actuation model of a garter knit actuation textile fabricated from variable stiffness smart material wire which takes all these factors into account. The states of operation are defined based on the mechanical loading of the textile, the transition between different material stiffness, and the paths followed to arrive at each state. Operational transitions between these states induce frictional forces (stick or slip) depending upon the state and path, which affect the actuation response. A load-extension model is derived for each state of a typical actuation cycle with respect to the unit cell of a single loop of the textile based on the stress-strain behavior of the active material with assumptions made to allow analytical tractability. Elastica theory and Euler-Bernoulli beam bending are used to capture the large deformations within a loop of wire. The resulting kinematic and kinetic relations for a single cell scale the load-extension behavior of the entire knit textile for each state, and provide analytical algebraic transcendental expressions for the net actuation motion as a function of the applied tensile load on the textile. The model was validated experimentally for a thermally actuated SMA garter knit prototype over a range of applied forces with good correlation. The two-dimensional analytical active knit model provides the ability to predict actuation motions for this large stroke, large force actuation architecture, enabling the design of active knitted architectures for a wide range of applications.

\section{Garter stitch architecture and operation}

Garter knits are capable of generating large strains beyond the base material capability because of their unique architecture and multi-state operation, which are both described in this section.

\subsection{Architecture}

The architecture of a knit is defined by the arrangement of knit and purl connections between interlaced loops of adjacent 


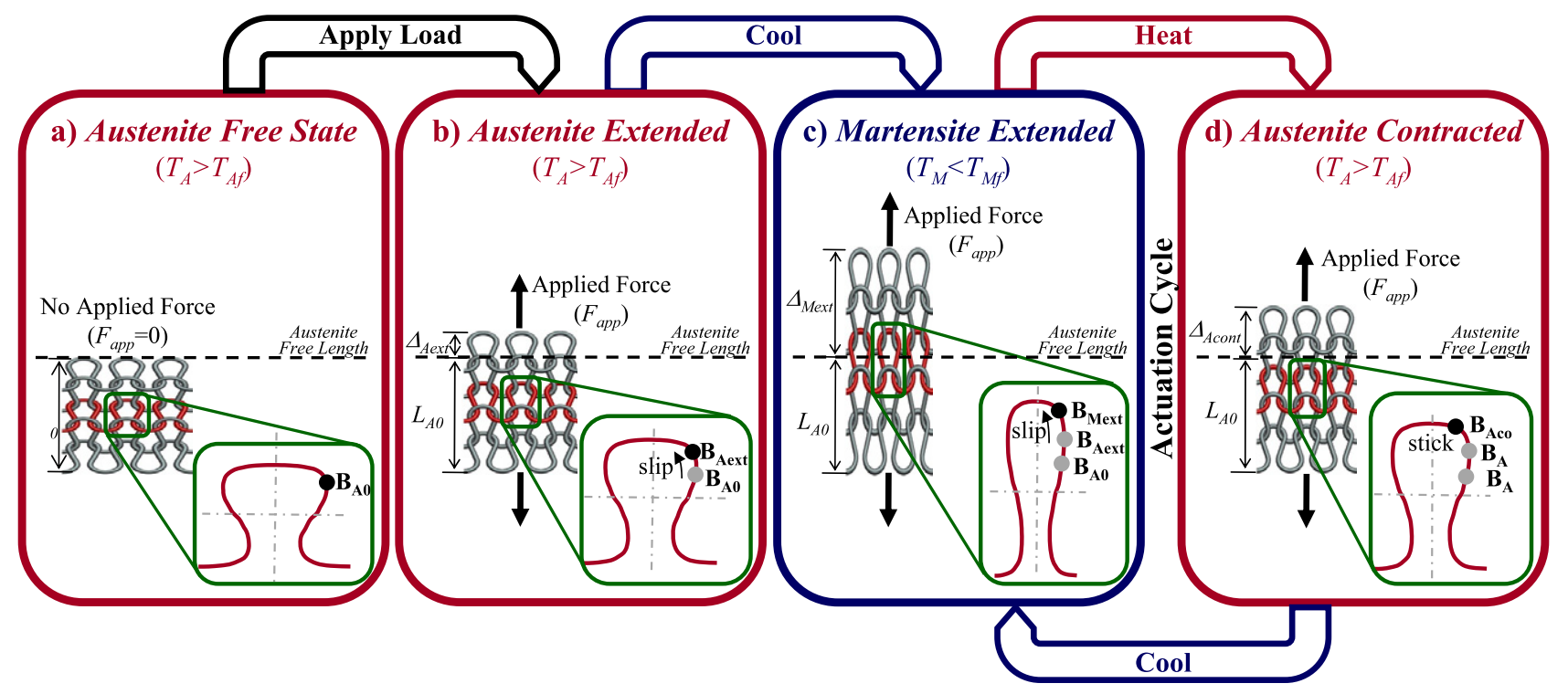

Figure 2. Active garter stitch states. (a) Austenite free state, (b) austenite extended state, (c) martensite extended state, and (d) austenite contracted state reached through thermal or mechanical operational transitions where a typical actuation cycle is alternates between the martensite extended and austenite contracted states.

courses (figure 1). A course is a row of knit loops, shown in red in figure 1 , which comprises a single strand of alternating (up and down) curved loops. A wale is a column of knit loops, the legs of each loop interlocking with the top of the next loop down the wale (i.e. in the next course). In a knit connection, the legs of the upper loop in a wale overlap the top of the lower loop in the same wale and interlace behind the sides of the loop, whereas in a purl connection the top of the lower loop overlap the legs of the upper loop which interlace behind the sides of the lower loop. The ordering of the knit and purl connections define the architecture where garter stitch, comprised of alternating courses of all knit and all purl connections, forms a planar textile symmetric about the front and back. The loops within the architecture provide large curvatures through which loops interlace with the adjacent loop making contact at the interlacing contact point, $\mathbf{B}$. The interlacing contact point may change position as adjacent loops slip relative to each other against friction, depending on the relative magnitudes of the loading and contact forces and the coefficient of friction between the wires. The garter knitis geometrically defined by its course height $(C)$, wale width $(W)$, loop length $(L)$, and fiber diameter $(d)$. The course height $(C)$ is the vertical distance between identical reference points of adjacent rows. The wale width $(W)$ is the horizontal distance between identical reference points on adjacent loops within a course. The loop length $(L)$ is the length along the centerline of the fiber of a single knit loop. The fiber diameter $(d)$ is the diameter of the foundational wire.

\subsection{Operation}

SMA garter knit textiles go through operational transitions from one state to another resulting in a change in length and stiffness of the textile. The operational transitions are initiated by either a change in thermal loading, inducing a material transition from flexible martensite to stiff austenite, or by changes in mechanical loading. During these operational transitions, different friction conditions occur between loops of the knit (stick or slip) depending on the loading and the initial state. The state of the textile is defined by the thermal loading (austenite or martensite), the mechanical loading and the loading path by which the textile arrived into that state (free, extended, or contracted). A typical actuation cycle (depicted in figure 2) is initiated from the austenite free state with an operational transition into the austenite extended state, and then cycles between the martensite extended state and the austenite contracted state. The length of the textile in each state, and therefore the motion produced by each operational transition, depends on four factors: (1) the state it is in, (2) the state it came from, (3) the magnitude of the mechanical loading, and (4) the inter-loop friction conditions experienced during operational transition into its state.

2.2.1. Austenite free state. A typical actuation cycle is initiated with the SMA garter knit textile in a heated state with no external loads. When the temperature of the SMA is raised above its austenite finish transition temperature, it makes a material transition from a soft martensite phase to a stiff austenite phase. In transitioning, deformations experienced in the martensite phase are recovered, producing the shape memory effect of SMA. While the material phase of SMA is a function of both temperature and stress, for modeling purposes, it is assumed that a complete thermal transition occurs throughout the wire and that the bending stresses are low enough such that no areas of stress induced martensite are developed. Because the martensite strains are recovered, the austenite free state provides a zero reference for computing material strains. In a knitted structure, however, the material is not actually in a zero-strain state since the wires, which come from the manufacturer trained to a straight shape, are bent in loops. When heated they attempt to return to their 
naturally straight configuration resulting in a relatively wide wale width, $W$, and a relatively short course height, $C$. Even though many internal stresses and strains exist, the garter knit textile length $L_{\mathrm{A} 0}$ in the austenite free state (figure 2(a)) is set as the zero deflection reference point for actuation.

\subsubsection{Austenite extended state. When a tensile load, $F_{\text {app }}$,} is applied to the textile in this heated state, the knit loops elongate, increasing the course height $C$, and lengthening the entire textile by a deflection $\Delta_{\text {Aext }}$, relative to the austenite free length, $L_{\mathrm{A} 0}$. During this operational transition into the austenite extended state (figure 2(b)), the adjacent loops of wire generally slip past each other from an initial point of contact $\mathbf{B}_{\mathrm{A} 0}$ along the loop to a final interlacing contact point $\mathbf{B}_{\text {Aext. }}$ The resulting friction resists the slip and therefore resists elongation of the textile, reducing the deflection $\Delta_{\text {Aext }}$ into this state.

2.2.3. Martensite extended state. When the textile is allowed to cool under load to a temperature below its martensite finish transition temperature, the textile makes a material transition into the martensite phase and becomes less stiff. It is assumed that the stiffness decreases as a result of a complete thermal transition as all the material cools below the martensite finish temperature. As a result, the course height of the loops elongates further into the martensite extended state (figure 2(c)) in which the textile is deflected by $\Delta_{\text {Mext }}$ relative to the austenite free length, where $\Delta_{\text {Mext }}>\Delta_{\text {Aext }}$. During this operational transition, adjacent loops slip further past each other from the austenite extended interlacing point of contact $\mathbf{B}_{\text {Aext }}$, to the martensite extended interlacing point of contact $\mathbf{B}_{\text {Mext }}$ such that friction again reduces the deflection experienced by the textile during this operational transition. The martensite extended state is the first of two states in the cyclic portion of the typical actuation cycle.

2.2.4. Austenite contracted state. The next state of the actuation cycle is entered when the material is heated under load to above its austenite finish transition temperature to the austenite phase. The resulting stiffening of the material causes the wires within each loop to attempt to straighten to their natural straight shape, reducing the course height, and inducing contraction of the textile to a length deflected from the austenite free length by a distance $\Delta_{\text {Acont }}$, where $\Delta_{\text {Mext }}>\Delta_{\text {Acont }}>\Delta_{\text {Aext }}$. During this operational transition into the austenite contracted state (figure 2(d)), friction between the loops opposes relative motion of the interlacing point of contact back downward toward the austenite extended interlacing point of contact $\mathbf{B}_{\text {Aext }}$. In most cases, the friction is observed to completely block slippage such that the austenite contracted interlacing point of contact $\mathbf{B}_{\text {Acont }}$ remains stuck at the martensite extended interlacing point of contact $\mathbf{B}_{\text {Mext }}$. Thus, even though the thermal and mechanical loading are identical in the austenite extended state and the austenite contracted state, the length in the austenite contracted state is longer than that of the austenite extended state due to the change in direction and stick-slip nature of the friction conditions experienced in arriving at each state.
Actuation against an applied force $F_{\text {app }}$ occurs cyclically between the austenite contracted state and the martensite extended state, where the net actuation deflection $\Delta_{\mathrm{ACT}}$ is equal to the difference between the martensite extended deflection and the austenite contracted deflection $\left(\Delta_{\mathrm{ACT}}=\right.$ $\left.\Delta_{\text {Mext }}-\Delta_{\text {Aext }}\right)$.

\section{Analytical garter stitch model}

An analytical state-based actuation model is derived for the garter knit architecture to predict the load-extension behavior of each state and net actuation motion as a function of the applied tensile load on the textile, thermal load and material phase, and path dependent friction. While several load-extension models exist for passive textile knits [62-82], they do not model the multi-state contractile actuation of active knits. Existing knit models are typically purely geometric and only capture small deformations, while engineering models that account for larger deformations only predict the load-extension behavior of a passive single-state knit. None of these existing models incorporate thermal and mechanical operational transitions from one state to another, and they all neglect the influence of the load path and the interlacing loop friction.

These passive knit models however, do provide a starting foundation for the modeling of the load-extension of individual states of an active knit. By modifying and combining existing passive knit models, particularly those developed by Hong [75] and Shanahan [74], a quasi-static analytical model can be developed that predicts the actuation of the active knit based on the state it is in, the state it came from, and the inter-loop friction experienced during the operational transition into the state. The required modifications include incorporating slipping and sticking between adjacent interlacing loops, enforcing compatibility of displacements and curvature at the interlacing contact point, and accounting for the change in stiffness between states.

The modeling approach uses a quarter of the knit loop as the unit cell of the knit architecture. Geometric relations are established for the unit cell taking advantage of the high level of symmetry within the structure to describe the position of key points and inflections within the cell. The reference austenite free state is modeled to establish the base geometry and loop shape, as well as the load-extension behavior of the austenite and martensite extended, and the austenite contracted states. For each state, the shape of the loop is established using elastica theory and Euler-Bernoulli beam bending to capture the large bending rotations using a small strain linear deflection approximation of the SMA wire in each phase. Equilibrium equations are developed for the loop including the stick or slip friction interaction between loops depending on the particular operational transition. The resulting governing differential equations are integrated along the loop using boundary conditions derived from geometry to provide a set of algebraic equations relating a set of three unknown loop state angles to the overall size of the cell. Kinetic relations for each state are formulated to relate the loading on each loop to its shape providing fully analytical 
transcendental equations from which the load-extension behavior of the unit cell is derived. The difference between the load-extension behavior of the martensite extended and austenite contracted states describes the actuation behavior of the unit cell. The unit cell properties are scaled by the numbers of courses and wales to provide the load-extension behavior and net actuation behavior of the entire knit textile.

\subsection{Assumptions}

To simplify the modeling approach and to enable analytical tractability, several assumptions about the fibrous active material and loop architecture are employed. The fiber is assumed to be a naturally straight, inextensible, incompressible, homogeneous, elastic rod that experiences the shape memory effect. The SMA wire is assumed to be trained to a straight shape that is recoverable upon heating at low load levels. While inextensibility is not traditionally associated with SMA wire because of its large axial extension and contraction capability, it is used in the knitted model because at typical load levels the extension of the knit is assumed to be governed by the change of shape in the knit loop caused by bending. The inextensibility assumption implies a constant diameter for the SMA wire.

The effective modulus of elasticity is phase dependent $\left(E_{\mathrm{A}}, E_{\mathrm{M}}\right)$, assuming the material fully transforms between phases as discussed in the operation section, and is derived from small strain limiting cases of the nonlinear stress-strain relationships. In reality, the bending curvatures of the SMA material may not allow complete transformation into the austenite phase and a portion of the material may be in the stress induced martensite phase. Also, while linear elastic assumptions apply well to the full austenite phase they only apply to the martensite phase for small strains. However, these simplified material assumptions are made to enable tractability of the analytical model, and they do apply to situations with relatively large loop to wire diameter ratios, therefore to more loosely knit architectures.

In addition to assumptions about the fibrous material several loop architecture assumptions have been implemented. Every loop in the knitted textile is assumed to have the same planar geometry with no out of plane behavior during deformation. Interlacing loops from adjacent courses are assumed to always be in contact and the central axes of these loops are separated by one wire diameter (figure 3 ). A simplified fiber reaction force, $R$, acting at a single point between the interlacing fibers perpendicular to the fiber axis is assumed.

\subsection{Geometric relations}

Geometry plays a crucial role in the development of an analytical model of active knitted material because it allows for the establishment of physical constraints between the known geometric parameters (course height $-C$, wale width $-W$, loop length $-L$, and wire diameter $-d$ ) and the unknown loop state angles $(\alpha, \beta$ and $\gamma$ ). The three angles (the angle of the reaction force $(R)$ between adjacent loops- $\beta$, the angle of the connecting leg at $\mathbf{A}-\alpha$, and the angle of force $P$ at $\mathbf{A}-\gamma)$ describe the geometric loop shape under different loads. The undetermined loop state angles vary during loading to allow for the direction of internal forces to change and slipping of the interlacing contact point during extension. The geometric constraints are developed by analyzing a unit cell of the knit architecture. The unit cell is one quarter of the knit loop and a quarter of the interlacing adjacent loop (figure 3). A complete knit loop can be assembled using symmetry by rotating and reflecting the unit cell about the $X$ and $Y$-axes defining the origin at this center of symmetry, $\mathbf{O}$, and matching the connection points at the top (or bottom) of the loop at $\mathbf{C}$ and at the end of the connecting leg at $\mathbf{A}$. Symmetry dictates the position and curvature at point $\mathbf{A}$. To ensure compatibility of displacements along the length of the wire $\mathbf{A}$ must be located in the bottom center of the unit cell at $(0, W / 4)$. Continuity requires $\mathbf{A}$ to be an inflection point acting along the angle $\alpha$, because the direction of the curvature of the wire in the knit loop changes therefore $\mathbf{A}$ cannot support a moment. From $\mathbf{A}$ the wire begins to curve upward until it reaches the interlacing contact point $\mathbf{B}$, the point along the central axis of the knit loop through which the resultant force between adjacent loops acts. To maintain symmetry, the line of action of the resultant force, $R$, which acts perpendicular to the tangent line at $\mathbf{B}$, must pass through the center of the unit cell, $\mathbf{D}$, located at $(W / 4, C / 2)$ and representing the point of interaction between adjacent loops, requiring $\mathbf{B}$ to lay a distance of $d / 2$ from $\mathbf{D}$ along an angle of $\beta-\pi / 2$. From $\mathbf{B}$, the foundational wire continues to curve toward the top of the knit loop, point $\mathbf{C}$, located at an unknown height along the left-most edge of the unit cell.

Four different geometric relations can be derived from the unit geometry. The first geometric constraint,

$$
\frac{W}{4}=X_{B}-\frac{d}{2} \cos \left(\beta-\frac{\pi}{2}\right),
$$

relates $X_{B}$, the $X$-coordinate of the interlacing contact point, $\mathbf{B}$, to $W / 4$, the $X$ position of point $\mathbf{A}$, using the assumption that the adjacent interlocking wires are in contact and incompressible, therefore separated by the wire diameter, $d$, along the line of action of $R$. Similarly, a second constraint,

$$
X_{B}-X_{A}=\frac{d}{2} \cos \left(\beta-\frac{\pi}{2}\right),
$$

couples the difference in the $X$-coordinates of the interlacing contact point and leg end, $X_{B}$ and $X_{\mathrm{A}}$, to the horizontal component of the distance between the loop interaction point, D, and half the wire diameter. Using the same assumptions as used for the development of wale width constraint (equation (1)), a third constraint,

$$
\frac{C}{2}=Y_{B}-\frac{d}{2} \sin \left(\beta-\frac{\pi}{2}\right),
$$

associates the $Y$-coordinate of point $\mathbf{B}$, with the vertical location of the center of the unit cell, $C / 2$, using the wire diameter, $d$, and angle, $\beta$. The final geometric constraint,

$$
\frac{L}{4}=s_{\mathrm{AB}}+s_{\mathrm{BC}},
$$




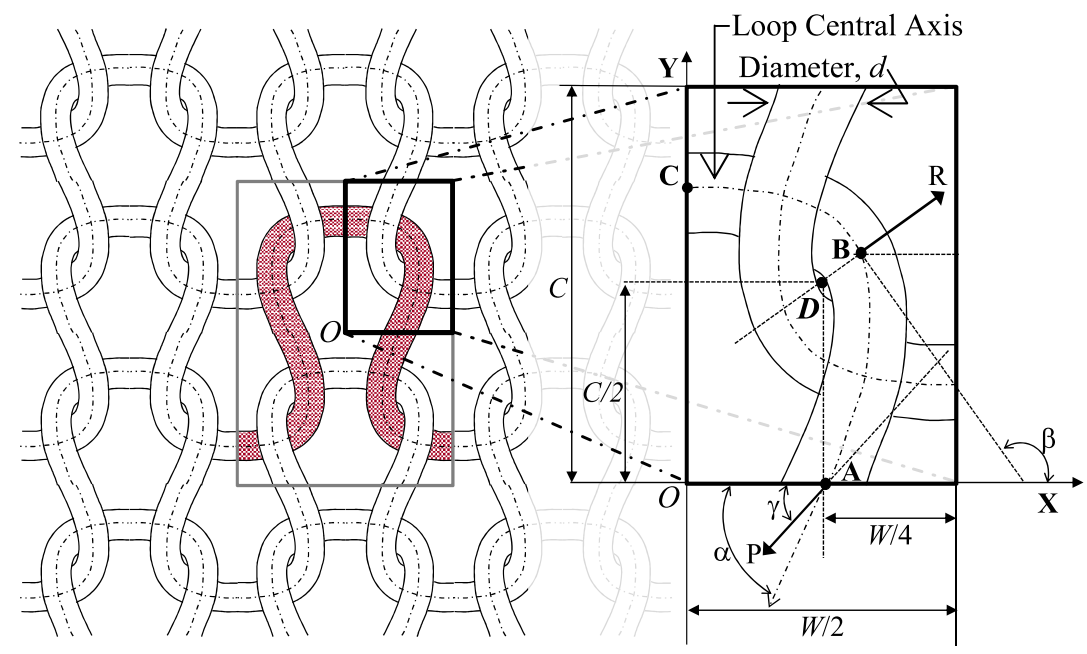

Figure 3. Knit unit cell. The knit unit cell is one quarter of a knit loop (shown in red) and is defined by geometric parameters (course height $-C$, wale width $-W$, loop length $-L$, wire diameter $-d)$ and unknown characteristic angles $(\alpha, \beta$, and $\gamma)$.

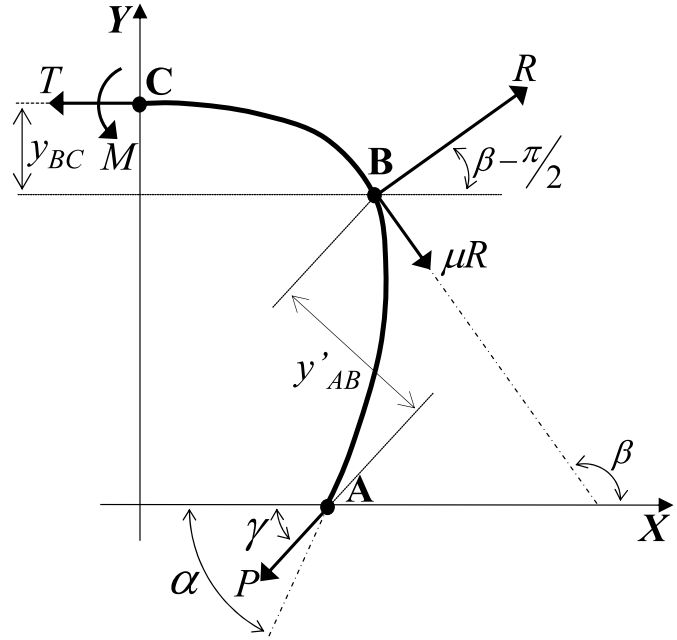

Figure 4. Garter knit unit cell free body diagram. The free body diagram includes the internal forces and moments- $T, R, \mu R, P$ and $M$ and the loop state angles $(\alpha, \beta$, and $\gamma)$.

is a compatibility equation relating the length of wire in the loop, $L$, to the combined length of segment $\mathbf{A B}, s_{\mathrm{AB}}$, and length of segment $\mathbf{B C}, s_{\mathrm{BC}}$, in this quarter knit loop established using the inextensibility assumption.

\subsection{Austenite free state (reference state)}

The austenite free state acts as a reference state upon which all further calculations are made. The state is developed by analyzing the force-equilibrium interactions and the governing differential equations for the segment of the loop above and below the interlacing contact point. The analysis allows for kinematic loop state equations to be derived in terms of measurable geometric parameters (course height $-C$, wale width $-W$, loop length $-L$, wire diameter $-d$ ) and unknown loop state angles $(\alpha, \beta$, and $\gamma)$ which can be solved to provide the loop state angles. Once the loop state angles are known, the corresponding kinetic information (internal forces) can be calculated. The kinematic and kinetic values provide a reference state for the extension and contraction of the knitted structure.

3.3.1. Force-equilibrium interactions. The free body diagram of the planar knit loop (figure 4) is separated into two segments at the interlacing contact point (B) by the resultant force, $R$, transmitted by the adjacent interlocking loop. A friction force, $\mu R$, acts perpendicular to $R$ at $\mathbf{B}$ in the positive $X$ and negative $Y$ direction to resist extension from the as-knit geometry which typically has more overlap and slack between courses. A single force $P$ acts on point $\mathbf{A}$ at an angle of $\gamma$ relative to the $X$-axis, which is an inflection point and cannot support a moment. At point $\mathbf{C}$, a horizontal force, $T$, acts in the negative $X$-direction and a bending moment, $M$, acts counterclockwise (no shear can be supported due to symmetry).

Force-equilibrium analysis in the $X$ and $Y$ forces and moments provides kinetic loop relationships. Force-equilibrium in the $X$-direction results in

$$
T=R(\sin (\beta)-\mu \cos (\beta))-P \cos (\gamma) .
$$

Equilibrium in the $Y$-direction produces

$$
R=\frac{-P \sin (\gamma)}{\mu \sin (\beta)+\cos (\beta)},
$$

which can be combined with the tension in the top of the loop from the $X$-direction equilibrium (equation (5)) to eliminate $R$ and give the relationship

$$
T=-P\left(\sin (\gamma) \frac{\sin (\beta)-\mu \cos (\beta)}{\cos (\beta)+\mu \sin (\beta)}+\cos (\gamma)\right) .
$$

For future simplification the substitution 
(a)

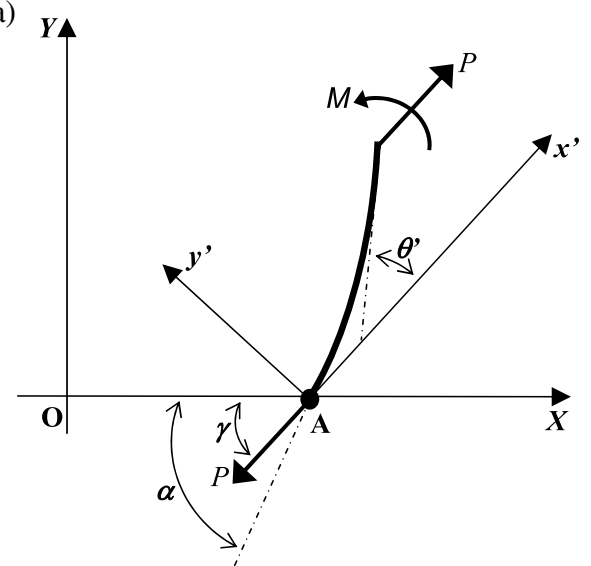

(b)

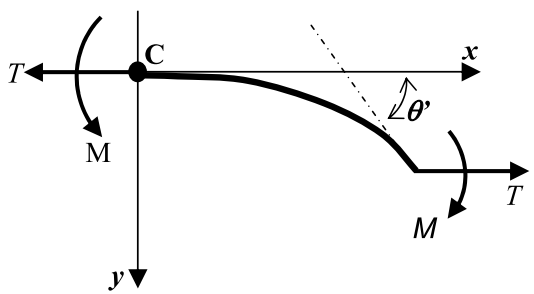

Figure 5. Loop segment free body diagrams. Free body diagrams for analysis of the governing differential equations of (a) segment AB and (b) segment BC.

$$
k_{1}=\sin (\gamma) \frac{\sin (\beta)-\mu \cos (\beta)}{\cos (\beta)+\mu \sin (\beta)}+\cos (\gamma)
$$

is made, where $k_{1}$ is a function of the geometric angles $\beta$ and $\gamma$ only.

The moment balance about $\mathbf{B}$ is

$$
M=P y_{\mathrm{AB}}^{\prime}-T y_{\mathrm{BC}}
$$

and can be simplified to

$$
M=P\left(y_{\mathrm{AB}}^{\prime}+k_{1} y_{\mathrm{BC}}\right)
$$

using the modified equation for the tension in the top of the loop (equation (7)), where $y_{\mathrm{AB}}^{\prime}$ is the perpendicular distance between the line of action of $P$ and $\mathbf{B}$ and $y_{\mathrm{BC}}$ is the vertical distance between $\mathbf{B}$ and the top of the loop, $\mathbf{C}$.

3.3.2. Analysis of governing differential equations. The governing differential equations describing the bending of the knit loop are developed using elastica theory and Euler-Bernoulli beam bending. Elastica theory is a theory of solid mechanics that accounts for large elastic rotations of structures during bending or buckling [77]. The use of elastica theory requires manipulation of the governing differential equations using the relations $\mathrm{d} x / \mathrm{d} s=\cos (\theta)$ and $\mathrm{d} y / \mathrm{d} s=$ $\sin (\theta)$ to obtain closed form solutions involving complex elliptic functions. Bending of the knit unit cell is analyzed in two sections, segments $\mathbf{A B}$ and $\mathbf{B C}$, while imposing continuity of displacements and slopes at $\mathbf{B}$.

3.3.2a. Lower loop segment $A B$. Segment $\mathbf{A B}$ is treated as an elastic rod pinned at $\mathbf{A}$ with an applied end load, $P$, and analyzed using a transformed coordinate system $\left(x^{\prime}, y^{\prime}\right)$ centered at A where $x^{\prime}$ is along but opposite the line of action of $P$ (figure 5(a)). The differential equation describing this portion of the loop is simply

$$
\frac{\mathrm{d} \theta^{\prime}}{\mathrm{d} s}=\frac{P y^{\prime}}{E_{\mathrm{A}} I}
$$

as determined from Euler-Bernoulli beam bending where $\theta^{\prime}$ is the angle with the $x^{\prime}$-axis, $s^{\prime}$ is the length along the loop, $E_{\mathrm{A}}$ is the phase dependent effective elastic modulus of the wire in the austenite phase, and $I$ is the second moment of inertia of the wire cross-section.

The governing differential equation for segment $\mathbf{A B}$ (equation (11)) is differentiated and the elastica relation $\mathrm{d} y / \mathrm{d} s=\sin (\theta)$ is used to obtain

$$
\frac{\mathrm{d}^{2} \theta^{\prime}}{\mathrm{d} s^{2}}=\frac{P}{E_{\mathrm{A}} I} \frac{\mathrm{d} y^{\prime}}{\mathrm{d} s}=\frac{P}{E_{\mathrm{A}} I} \sin \left(\theta^{\prime}\right) .
$$

This equation is multiplied by $\mathrm{d} \theta / \mathrm{d} s$ and rearranged, giving

$$
\frac{\mathrm{d}}{\mathrm{d} s}\left[\frac{1}{2}\left(\frac{\mathrm{d} \theta^{\prime}}{\mathrm{d} s}\right)^{2}+\frac{P}{E_{\mathrm{A}} I} \cos \left(\theta^{\prime}\right)\right]=0,
$$

which can be integrated to determine the length of the wire, $s_{A B}$, using the boundary conditions derived from geometry and the fact that point $\mathbf{A}$ is an inflection point

$$
\begin{gathered}
\left.\theta^{\prime}\right|_{s=0}=\alpha-\gamma, \\
\left.\frac{\mathrm{d} \theta^{\prime}}{\mathrm{d} s}\right|_{s=0}=0, \quad \text { and } \\
\left.\theta^{\prime}\right|_{s=s_{\mathrm{AB}}}=\beta-\gamma .
\end{gathered}
$$

Two substitutions,

$$
\begin{aligned}
& u=\cos \left(\frac{\theta^{\prime}}{2}\right) \quad \text { and } \\
& z=\arcsin \left(\frac{u}{\cos \left(\frac{\alpha-\gamma}{2}\right)}\right),
\end{aligned}
$$

are useful to manipulate the integral into a closed form elliptic integral describing the length of segment $\mathbf{A B}$,

$$
s_{\mathrm{AB}}=\sqrt{\frac{E_{\mathrm{A}} I}{P}} \hat{F}\left(\varepsilon_{1}, \varphi_{1 \mathrm{~B}}\right),
$$

where

$$
\varepsilon_{1}=\cos \left(\frac{\alpha-\gamma}{2}\right)
$$




$$
\varphi_{1 \mathrm{~B}}=\arcsin \left(\cos \left(\frac{\beta-\gamma}{2}\right) / \cos \left(\frac{\alpha-\gamma}{2}\right)\right),
$$

and

$$
\hat{F}\left(\varepsilon_{1}, \varphi_{1 \mathrm{~B}}\right)=F\left(\varepsilon_{1}, \pi / 2\right)-F\left(\varepsilon_{1}, \varphi_{1 \mathrm{~B}}\right)
$$

given $F\left(\varepsilon_{1}, \pi / 2\right)$ is a complete elliptic integral of the first kind and $F\left(\varepsilon_{1}, \phi_{1 \mathrm{~B}}\right)$ is an incomplete elliptic integral of the first kind. The curvature, $\mathrm{d} \theta / \mathrm{d} s$, at any point along the length of segment $\mathbf{A B}$ is found by differentiating the length along the loop (the general form of equation (19)) with respect to $\theta$ to obtain the equation

$$
\left.\frac{\mathrm{d} \theta}{\mathrm{d} s}\right|_{s=s_{\mathrm{BC}}}=2 \varepsilon_{1} \sqrt{\frac{E_{\mathrm{A}} I}{P}} \cos \left(\varphi_{1 \mathrm{~B}}\right) .
$$

Integrating the combination of the elastica assumptions and the curvature relationship (equation (23)) the $x^{\prime}$ and $y^{\prime}$ coordinates for loop segment $\mathbf{A B}$ are determined to be

$$
\begin{gathered}
x_{\mathrm{AB}}^{\prime}=\sqrt{\frac{E_{\mathrm{A}} I}{P}}\left\{\hat{F}\left(\varepsilon_{1}, \varphi_{1 \mathrm{~B}}\right)-2 \hat{E}\left(\varepsilon_{1}, \varphi_{1 \mathrm{~B}}\right)\right\} \\
y_{\mathrm{AB}}^{\prime}=2 \sqrt{\frac{E_{\mathrm{A}} I}{P}} \varepsilon_{1} \cos \left(\varphi_{1 \mathrm{~B}}\right),
\end{gathered}
$$

where

$$
\hat{E}\left(\varepsilon_{1}, \varphi_{1 \mathrm{~B}}\right)=E\left(\varepsilon_{1}, \pi / 2\right)-E\left(\varepsilon_{1}, \varphi_{1 \mathrm{~B}}\right),
$$

and $E\left(\varepsilon_{1}, \pi / 2\right)$ is a complete elliptic integral of the second kind and $E\left(\varepsilon_{1}, \phi_{1 \mathrm{~B}}\right)$ is an incomplete elliptic integral of the second kind. Transforming the coordinate system from the local $x^{\prime}, y^{\prime}$ to the global $X, Y$ through the angle $\gamma$ results in

$$
\begin{gathered}
X=\frac{W}{4}+x^{\prime} \cos (\gamma)-y^{\prime} \sin (\gamma) \quad \text { and } \\
Y=x^{\prime} \sin (\gamma)+y^{\prime} \cos (\gamma) .
\end{gathered}
$$

The analysis of the lower loop segment AB provides half the fundamental equations used in determining the loop state angles.

3.3.2b. Upper loop segment BC. Segment BC is treated as an elastic rod clamped at $\mathbf{C}$ with a horizontal load, $T$, and an applied moment, $M$, while $\mathbf{B}$ is free to move (figure 5(b)) but must satisfy continuity conditions with $\mathbf{A B}$. Segment $\mathbf{B C}$ is analyzed using the transformed coordinate system $(x, y)$ where $x$ is shifted vertically from $X, \mathbf{C}$ acts as the origin, and $y$ acts in the negative $Y$ direction. The differential governing equation for segment $\mathbf{B C}$ is given by

$$
\frac{\mathrm{d} \theta}{\mathrm{d} s}=\frac{T y+M}{E_{\mathrm{A}} I},
$$

where $\theta$ is the angle between the $x$-axis and the tangent line to the loop. The modified equation for the tension in the top of the loop (equation (7)) and the moment at the top of the loop (equation (10)) are substituted into the governing differential equation for segment BC (equation (29)), which is differentiated and the elastica relation is employed resulting in the manipulated equation

$$
\frac{\mathrm{d}}{\mathrm{d} s}\left[\frac{1}{2}\left(\frac{\mathrm{d} \theta}{\mathrm{d} s}\right)^{2}+\frac{T}{E_{A, M} I} \cos (\theta)\right]=0 .
$$

The differentiated governing differential equation (equation (30)) can be integrated to determine the length of the wire, $s_{\mathrm{BC}}$, in segment $\mathbf{B C}$ using the geometric boundary conditions

$$
\begin{gathered}
\left.\theta\right|_{s=0}=0, \\
\left.\theta\right|_{s=s_{\mathrm{BC}}}=\pi-\beta, \quad \text { and } \\
\left.\frac{\mathrm{d} \theta}{\mathrm{d} s}\right|_{s=s_{\mathrm{BC}}}=2 \varepsilon_{1} \sqrt{\frac{E_{\mathrm{A} I}}{P}} \cos \left(\varphi_{1 \mathrm{~B}}\right),
\end{gathered}
$$

where the boundary condition for the curvature at the interlacing contact point B (equation (33)) enforces continuity of wire curvature at $\mathbf{B}$. Employing the substitutions

$$
\begin{gathered}
u=\cos \left(\frac{\theta}{2}\right) \quad \text { and } \\
z=\arccos (u),
\end{gathered}
$$

this integration results in an equation describing the length of segment BC,

$$
s_{\mathrm{BC}}=\sqrt{\frac{E_{\mathrm{A}} I}{P}} \sqrt{\frac{2}{k_{1}+k_{2}}} F\left(\varepsilon_{2}, \varphi_{2 B}\right),
$$

where

$$
\begin{gathered}
\varphi_{2 B}=\frac{\pi-\beta}{2}, \\
\varepsilon_{2}=\sqrt{\frac{2 k_{1}}{k_{1}+k_{2}}}, \quad \text { and } \\
k_{2}=2 \varepsilon_{1}^{2} \cos ^{2}\left(\varphi_{1 \mathrm{~B}}\right)+k_{1} \cos (\beta),
\end{gathered}
$$

and $F\left(\varepsilon_{2}, \phi_{2 B}\right)$ is an incomplete elliptic integral of the first kind. The $x$ and $y$ coordinates of $\mathbf{B}$ can be determined to be

$$
\begin{aligned}
x_{\mathrm{BC}}= & \sqrt{\frac{E_{\mathrm{A}} I}{P}} \sqrt{\frac{2}{k_{1}+k_{2}}}\left(\frac{2}{\varepsilon_{2}^{2}} E\left(\varepsilon_{2}, \varphi_{2 B}\right)\right. \\
& \left.+\left(1-\frac{2}{\varepsilon_{2}^{2}}\right) F\left(\varepsilon_{2}, \varphi_{2 B}\right)\right)
\end{aligned}
$$

and

$y_{\mathrm{BC}}=\sqrt{\frac{E_{\mathrm{A}} I}{P}} \sqrt{\frac{2}{k_{1}+k_{2}}}\left(\frac{-2}{\varepsilon_{2}^{2}}\left(\sqrt{1-\varepsilon_{2}^{2} \cdot \sin ^{2}\left(\varphi_{2 B}\right)}-1\right)\right)$

using the elastica criteria. No coordinate transformation is needed for the $x$-coordinate but the equation

$$
Y=Y_{\mathrm{AB}}+\left(y_{\mathrm{BC}}-y\right)
$$

transforms any local coordinate $y$ into a global coordinate $Y$.

3.3.3. Kinematic relations. The kinematic equations developed during the analysis of the knit unit cell provides 
a set of nonlinear algebraic loop state equations that relate the initially measurable geometric parameters: wale width $(W)$, course height $(C)$, wire diameter $(d)$, and loop length $(L)$, to the unknown loop state angles. During the austenite free state, when no external load is applied to the knit unit cell, the loop state equations can be solved to obtain the loop state angles and the internal forces can be calculated.

The geometric constraints (equations (1)-(4)) can be modified with the new definitions of the coordinates and loop length segments (equations (19), (27), (28), (36) and (41)) in terms of the unknown loop state angles $(\alpha, \beta$ and $\gamma)$ and manipulated to eliminate the unknown force $P$ to obtain the three loop state equations:

$$
\begin{gathered}
\frac{L}{W}=\frac{C_{4}}{C_{1}-C_{3}}, \\
\frac{L}{C}=\frac{2 C_{4}}{C_{2}+C_{3} \cot (\beta)}, \\
\frac{L}{d}=\frac{2 C_{4} \sin (\beta)}{C_{3}},
\end{gathered}
$$

which relate the geometric parameters $(W, C, L$, and $d$ ), which can be measured from any loop in the austenite free state of the knit textile, to the unknown loop state angles. The three algebraic loop state equations provide a set of simultaneous nonlinear equations where the $C_{i}$ s are only functions of $\alpha, \beta$ and $\gamma$ and are given by

$$
C_{1}=\sqrt{\frac{2}{k_{1}+k_{2}}}\left[\frac{2}{\varepsilon_{2}^{2}} E\left(\varepsilon_{2}, \varphi_{2 B}\right)+\left\{1-\frac{2}{\varepsilon_{2}^{2}}\right\} F\left(\varepsilon_{2}, \varphi_{2 B}\right)\right],
$$$$
C_{2}=\sin (\gamma)\left\{f\left(\varepsilon_{1}, \varphi_{1 \mathrm{~B}}\right)-2 e\left(\varepsilon_{1}, \varphi_{1 \mathrm{~B}}\right)\right\}
$$$$
+2 \varepsilon_{1} \cos (\gamma) \cos \left(\varphi_{1 \mathrm{~B}}\right) \text {, }
$$$$
C_{3}=\cos (\gamma)\left\{f\left(\varepsilon_{1}, \varphi_{1 \mathrm{~B}}\right)-2 e\left(\varepsilon_{1}, \varphi_{1 \mathrm{~B}}\right)\right\}
$$$$
-2 \varepsilon_{1} \sin (\gamma) \cos \left(\varphi_{1 \mathrm{~B}}\right), \quad \text { and }
$$

$C_{4}=f\left(\varepsilon_{1}, \varphi_{1 \mathrm{~B}}\right)+\sqrt{\frac{2}{k_{1}+k_{2}}} F\left(\varepsilon_{2}, \varphi_{2 B}\right)$.

By making the initial material assumptions, the set of nonlinear differential equations was reduced to a set of three simultaneous transcendental algebraic equations. Using the measured geometric parameters, the system of loop state equations (equations (43)-(45)) can be solved numerically to determine the unknown loop state angles $(\alpha, \beta$ and $\gamma)$ that describe the unloaded loop. The kinematic analysis of the loop state equations provides the unknown geometric angles describing the loop in the austenite free state.

\subsubsection{Kinetic relations. Once the loop state angles are} known the load at $\mathbf{A}$ can be determined using the geometric length constraint and the definition of $C_{4}$ (equations (4) and (49)) to be

$$
P_{A}=\frac{16 E_{\mathrm{A}} I C_{4}^{2}}{L^{2}}
$$

acting at an angle $\gamma$ to the $X$ axis. The load, $P_{\mathrm{A}}$, is the internal force at point $\mathbf{A}$ that maintains the loop shape.The initial tension in the top of the loop, $T_{0}$, can be determined using the equation of the tension in the top of the loop (equation (7)) and the initial $Y$ component of the force $P_{\mathrm{A}}, P_{y 0}$, can be calculated using the relationship, $P_{y 0}=P_{A} \sin (\gamma)$. While there are no external forces acting on the knit loop, internal tensions exist within the loop.

\subsection{Austenite and martensite extended states}

The development of the equations representing the austenite and martensite extended states parallels that of the austenite free state. The force-equilibrium interactions are identical with friction still opposing extension. The governing differential equations for the austenite extended state are equivalent to those of the austenite free state, while the governing differential equations for the martensite extended state are the same except $E_{\mathrm{A}}$ (the austenite elastic modulus) is replaced by $E_{\mathrm{M}}$ (the representative martensite elastic modulus). However, with the kinematic relations an additional loop state equation is developed because the relationship between the wale width and course height must be determined as the textile is extended. The kinetic relations differ with the inclusion of an external load in the expression of the load $P$ at the leg of the unit cell and the development of a load-deflection relation for both the unit cell and the entire textile. The kinetic and kinematic relations for the austenite and martensite extended states are developed for austenite as an illustration, where the martensite relations differ only by the material modulus of elasticity $E_{\mathrm{M}}$.

3.4.1. Kinematic relations. The characteristic loop state angles must be calculated for the extended state under applied loads as the textile extends, requiring an additional constraint because the wale width, $W$, narrows from the austenite free state as the course height, $C$, lengthens. The loop length, $L$, and the diameter, $d$, remain constant during extension therefore the wire diameter/loop length loop state equation (equation (45)) can still be used. The wale width/loop length loop state equation (equation (43)) establishes a relationship to the load dependent loop state angles $(\alpha, \beta$ and $\gamma)$. A third equation is necessary to solve for these unknown angles. This is derived from the assumption that the tension in the top of the loop, $T$, remains constant from the austenite free state as increases in the horizontal component of $R$ are taken up through $P$ due to symmetry thus there can be no change in horizontal loading [75] of the unit cell. Constant horizontal loop tension produces the relationship

$$
T_{A}=\frac{-16 E_{\mathrm{A}} I C_{4}^{2} k_{1}}{L^{2}},
$$

as derived from the equation for the tension in the top of the loop (equation (7)) and the equation for the load, $P$, applied at $\mathbf{A}$ (equation (50)). In the constraint for the tension at the top of the loop (equation (51)) the tension, $T_{\mathrm{A}}$, is known from the austenite free state, and the loop length $(L)$, the moment of inertia $(I)$, and the elastic modulus $E_{\mathrm{A}}$ are known values based on the phase of the material and the measured geometric properties, while $C_{4}$ and $k_{1}$ are geometric functions of the 
unknown loop state angles. The set of three simultaneous transcendental algebraic equations for the extended state loop state (equations (45)-(51)) can be solved to obtain the set of loop state angles that describe the loop shape for that extended state.

3.4.2. Kinetic relations. The loop state angles are used to calculate the applied force and the corresponding deflection of the knit unit cell from the austenite free state. The kinetic behavior of the textile is calculated by scaling the force on the knit loop by the number of wales in the textile while the deflection is scaled by the number of courses.

The applied force distributed on each unit cell, $F_{\mathrm{UC}}$, is the difference between the initial internal force component acting in the $Y$-direction, $P_{y 0}$, and the $Y$-component of $P, P_{y}$, in the extended state and is calculated as

$$
F_{\mathrm{UC}}=P_{y}-P_{y 0}
$$

after using the kinetic equation (equation (50)) to determine the force, $P$, acting at $\mathbf{A}$ for the extended loop. The associated deflection is calculated using the geometric constraint between the course height and the wire diameter, the global $Y$-coordinate transformation, and the definition of $C_{2}$ (equations (3), (28) and (47)) as

$$
\begin{aligned}
\delta_{\mathrm{A}, \text { ext }}= & 2\left(\sqrt{\frac{E_{\mathrm{A}} I}{P_{A}}} C_{2}-\sqrt{\frac{E_{\mathrm{A}} I}{P_{A}} C_{20}}\right) \\
& +d\left(\cos (\beta)-\cos \left(\beta_{0}\right)\right),
\end{aligned}
$$

which represents the difference between the initial course height and the extended course height under known applied load. The force-deflection relationship is comprised of transcendental expressions for the applied loop force $\left(F_{\mathrm{UC}}\right)$ and the corresponding deflection $\left(\delta_{\mathrm{A}, \mathrm{ext}}\right)$ which are parameterized by the wale width $W$. The set of loop state angles $(\alpha, \beta$, and $\gamma)$ obtained following the kinematic analysis are used in equations (52) and (53) to compute the force and deflection relationship as the width of the wale decreases.

The model of the knitted textile is developed by scaling the force-deflection model of the planar knit unit cell. It was assumed during the development of the knit model that a knit loop was made up of four identical unit cells rotated and reflected about the $X$ and $Y$ axes and a knitted textile was made of a matrix of knit loops. The total force in a knitted loop, $F_{\text {loop}}$, is twice that of the unit cell, $F_{\text {loop }}=2 F_{\mathrm{UC}}$, because there are two knit legs supporting the same force in each knit loop. The total force in a knitted textile is the addition of the force through all of the loops in the row or wale, $N_{\mathrm{W}}$, and can be expressed as $F_{\text {app }}=N_{\mathrm{W}} F_{\text {loop }}$. The extension of the unit cell is the same as the extension of a knitted loop therefore the extension of the textile is total extension through each course, $N_{\mathrm{C}}$, and can be calculated using the relationship $\Delta=N_{\mathrm{C}} \delta_{\text {Aext }}$. The load-extension of the knit unit cell is thus scaled to predict the load-extension of the entire knitted textile.

\subsection{Austenite contracted state}

The development of the equations for the austenite contracted state parallels that of the austenite free state. the force-equilibrium interactions are similar but sticking at the interlacing contact point $(\mathbf{B})$ is assumed. The governing differential equations for the austenite contracted state are exactly the same as the equations for the austenite free state. However, the kinematic relations require knowledge of the martensite extended state to enforce the assumption that no slipping occurs during actuation under a constant load, leading to two new loop state equations involving the applied load and constant segment length. The kinetic relations for the unit cell and the entire textile produce the load-extension relationship for the austenite contracted state while actuator displacement is provided by subtracting the austenite contracted state from the martensite extended state.

3.5.1. Kinematic relations. During actuation the knit loops widen as the martensite bending strain in the loop segments is recovered because the material stiffens during the transition into austenite. The interlacing contact point acts as a pin joint due to sticking between adjacent loops. The applied load in the $Y$-direction remains constant for the austenite and martensite extended and the austenite contracted states since the actuation cycle occurs against a constant applied load,

$$
F_{\mathrm{UC}}=P_{\mathrm{yUC}, \mathrm{Aext}}=P_{\mathrm{yUC}, \mathrm{Mext}}=P_{\mathrm{yUC}, \text { Acont }},
$$

where $P_{\mathrm{yUC}}$, Aext is the load applied to extend the unit cell in the austenite phase, $P_{\mathrm{yUC}}$,Mext is the load applied to extend the unit cell in the martensite phase, and $P_{\mathrm{yUC}}$, Acont is the load under which the unit cell is actuated to the austenite contracted state from the martensite extended state. The actuation force, $P_{\text {yUC,Acont }}$, can be broken into two components,

$$
\begin{aligned}
& P_{\mathrm{yUC}, \text { Acont }}=P_{\mathrm{y}, \text { Acont }}-P_{y 0, \text { Acont }} \\
& \quad=P_{\text {Acont }} \sin \gamma_{\text {Acont }}-P_{\text {Acont } 0} \sin \gamma_{\text {Acont } 0} .
\end{aligned}
$$

Combining and rearranging the above equations in terms of known forces and angles gives

$$
P_{\text {Acont }} \sin \gamma_{\text {Acont }}=P_{\text {yUC, } \text { Mext }}+P_{y 0, \text { Acont }},
$$

where the applied load for the martensite extended state, $P_{\mathrm{yUC}, \text { Mext }}$, and the Y-component of the austenite contracted state, $P_{y 0 \text {,Acont }}$, are known. The internal force acting on the knit unit cell at point $\mathbf{A}, P_{\text {Acont }}$, can be calculated using equation (50), resulting in

$$
\frac{16 E_{\mathrm{A}} I C_{4}^{2}}{L^{2}} \sin \gamma_{\text {Acont }}=P_{\text {yapp, Mext }}+P_{y 0, \text { Acont }},
$$

which is a loop state equation for the new contracted loop geometry.

The geometric constraint that the segment lengths must add to a quarter of the measured loop length (equation (4)) is given by the same loop state equation as was used for the extended states, (equation (45)), which is the second loop state equation for the austenite contracted state.

The third loop state equation is developed using the sticking assumption, which requires the lengths of segment $\mathrm{AB}$ and $\mathbf{B C}$ to remain constant during contraction. Since the previous constraint requires lengths to add to a quarter of the measured loop length only one segment $\left(s_{\mathrm{AB}}\right.$ or $\left.s_{\mathrm{BC}}\right)$ can be 
held constant without over-defining the system. Segment AB is held constant between the martensite extended and austenite contracted giving the relationship

$$
\frac{s_{\mathrm{AB}}}{L}=\frac{\hat{F}_{\mathrm{AB}}}{4 C_{4}} .
$$

Equations (55)-(58) are the loop state equations for the austenite contracted state and are expressed in terms of measured geometric parameters previously determined from the martensite extended and austenite free state, and the unknown loop state angles. The set of three simultaneous algebraic transcendental equations can be solved to determine the shape of the knit loop in the austenite contracted state.

3.5.2. Kinetic relations. The actuation force is the same as the force applied to the unit cell, $P_{\mathrm{yUC}}$,Acont and is given by $F_{\mathrm{UC}}$. The corresponding deflection from the austenite free state is provided by

$$
\begin{aligned}
\delta_{\mathrm{A}, \mathrm{cont}}= & 2\left(\sqrt{\frac{E_{\mathrm{A}} I}{P_{A}}} C_{2}-\sqrt{\frac{E_{\mathrm{A}} I}{P_{A}}} C_{20}\right) \\
& +d\left(\cos (\beta)-\cos \left(\beta_{0}\right)\right)
\end{aligned}
$$

the same equation used for the austenite and martensite extended states. The actuation displacement of the knit unit cell is the difference between the martensite extended and the austenite contracted deflections,

$$
\delta_{\text {act }}=\delta_{\mathrm{M}, \mathrm{ext}}-\delta_{\mathrm{A}, \mathrm{cont}} .
$$

\subsection{Textile actuation prediction}

Prediction of the overall knit textile actuation from the unit cell model is done by scaling the force and actuation displacement of the unit cell in the austenite and martensite extended states by the number of courses (rows) and wales (columns) in the textile. The total applied force under which the knit textile is actuating is $F_{\text {app }}=2 N_{\mathrm{W}} F_{\mathrm{UC}}$, while the total actuator displacement of the knitted textile is $\Delta_{\text {act }}=N_{C} \delta_{\text {act }}$. The modeling procedure presented in this section produces an analytical model that captures the kinematic and kinetic behavior of each state experienced by this complex actuation architecture.

\section{Garter stitch experimental validation}

Experiments were conducted to understand the behavior of this new architectural actuation approach and assess the model's ability to predict the load-extension of each state and the net actuation. The model predictions were calculated in MATLAB using the measured initial geometric parameters of the knit prototype from the austenite free state (course height $-C$, wale width $-W$, wire diameter- $d$, and loop length $-L)$ and material properties $\left(E_{\mathrm{A}}\right.$ and $\left.E_{\mathrm{M}}\right)$. The model prediction was compared to the experimental results for the load-extension curves in the austenite and martensite extended states, the austenite contracted state, and also for net actuation between the martensite extended state and the austenite contracted state.

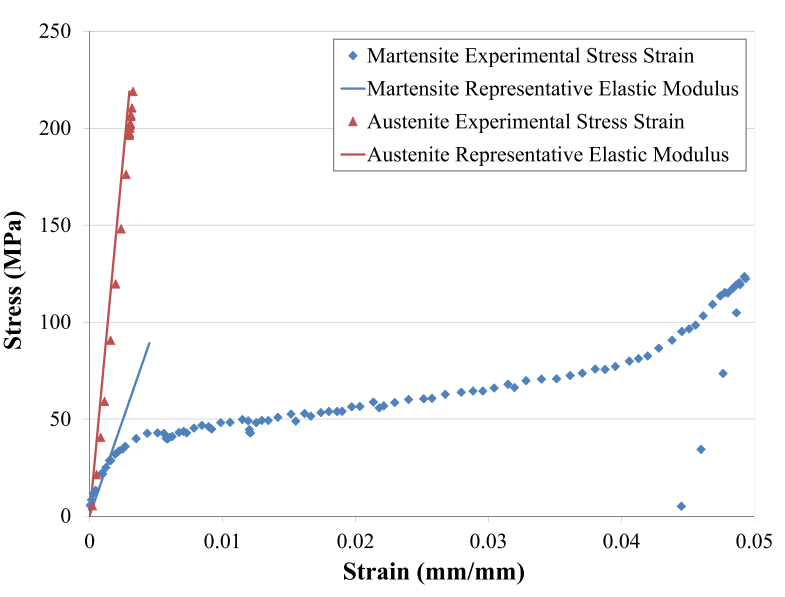

Figure 6. Experimental material properties of SMA wire. The material properties are represented with linear elastic austenite and martensite moduli.

\subsection{SMA material properties}

The material properties of the Flexinol $^{\circledR}$ wire were experimentally determined before validation of the analytical model. The austenite force-deflection curve for straight Flexinol ${ }^{\circledR} 70^{\circ} \mathrm{C}$ wire was generated by electrically heating the straight wire above the material's austenite finish temperature then measuring the load on the wire during axial elongation. Upon mechanical unloading, the wire returned to the austenite free length and wire was cooled to $20^{\circ} \mathrm{C}$ (below the martensite finish temperature). The martensite force-deflection curve was generated by measuring the load on the wire during wire extension at this lower temperature. This process produced two stress-strain curves (figure 6): a nearly linear austenite curve and a less stiff martensite curve with a nearly constant stress plateau. Linear elastic approximations of the stress-strain curves in the two phases were used, because the model assumes small strains during bending of the loops. The austenite elastic modulus was found to be $E_{\mathrm{A}}=73 \mathrm{GPa}$ while the representative martensite modulus, taken from the initial low-strain slope, was $E_{\mathrm{M}}=$ $19.8 \mathrm{GPa}$. These values are within the ranges of published elastic modulus values which vary from 70 to $75 \mathrm{GPa}$ for austenite and 18-28 GPa for martensite [83, 84].

\subsection{Active knit prototype}

A knit prototype was fabricated by hand knitting with 8 mil diameter, $d$, Dynalloy Flexinol ${ }^{\circledR} \quad 70^{\circ} \mathrm{C}$ shape memory alloy wire for 15 courses and 10 wales using $5.5 \mathrm{~mm}$ diameter knitting needles, $D$ (table 1, figure 7). The reference length and width of the prototype in the austenite free state were measured to be $L_{\mathrm{A} 0}=22.6 \mathrm{~mm}$ and $W_{\text {totalA } 0}=85.0 \mathrm{~mm}$. The average course height, $C$, was determined by dividing the total prototype length by the total number of courses $\left(N_{\mathrm{C}}=15\right)$ resulting in a course height of $1.5 \mathrm{~mm}$. Similarly, the wale width, $W$, was calculated by dividing the prototype width by the number of wales, $\left(N_{\mathrm{W}}=10\right)$, for a wale width of $8.5 \mathrm{~mm}$. The total wire length in the knit prototype was determined 
Table 1. Garter stitch prototype geometric parameters. Geometric parameters $\left(d, D, N_{\mathrm{C}}, N_{W}\right)$ used to create garter stitch knit and the measured austenite free state textile and unit cell geometries $\left(L_{\mathrm{A} 0}, W_{\mathrm{totalA} 0}, C_{0}, W_{0}\right.$, and $\left.L\right)$.

\begin{tabular}{lllllllll}
\hline$d(\mathrm{mil})$ & $D(\mathrm{~mm})$ & $N_{\mathrm{C}}$ & $N_{W}$ & $L_{\mathrm{A} 0}(\mathrm{~mm})$ & $W_{\text {totalA0 }}(\mathrm{mm})$ & $C_{0}(\mathrm{~mm})$ & $W_{0}(\mathrm{~mm})$ & $L(\mathrm{~mm})$ \\
8.0 & 5.5 & 15 & 10 & 22.6 & 85.0 & 1.5 & 8.5 & 20.2 \\
\hline
\end{tabular}

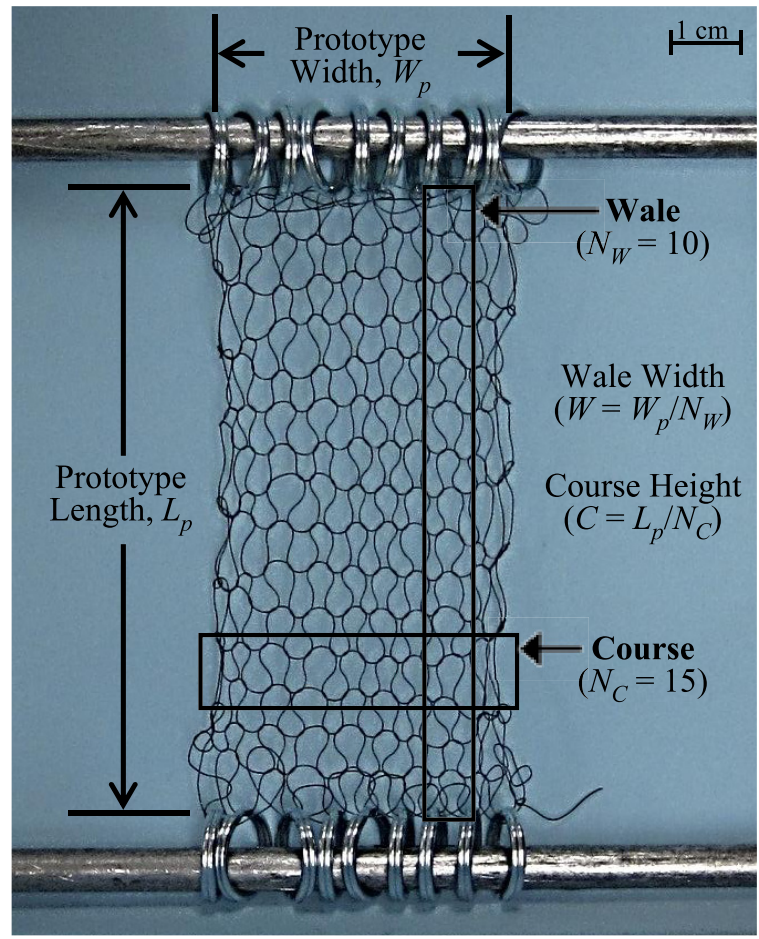

Figure 7. Garter stitch active knit prototype. Picture of the garter stitch active knit prototype in the martensite extended state under a $1 \mathrm{~N}$ applied load labeled with prototype geometric parameters.

post-knitting by comparing the prototype weight to the weight of a single wire of known length with the same diameter, then the loop length, $L$, of a single knit loop was calculated by dividing the total length by the total number of loops in the prototype $\left(N_{\mathrm{C}} \times N_{\mathrm{W}}\right)$, resulting in a knit loop length of $20.2 \mathrm{~mm}$.

\subsection{Experimental method}

The experimental set up depicted in figure 8 was utilized for all the garter experiments. The prototype was mounted to the experimental setup in its martensite free state to parallel horizontal smooth rails at the top and bottom of the prototype which moved orthogonally on linear bearings along parallel guide rails that spanned the length of the prototype. Free lateral (wale-wise) contraction during longitudinal (course-wise) prototype extension was permitted by a series of steel rings attaching the knit actuator to the smooth rails. Free lateral motion is critical to maintain free boundary conditions and uniform deformations of all the loops within the prototype as assumed by the model.

The prototype was run through a thermo-mechanical cycle (figure 2) in an Envirotronics EnviroFLX300 environmental chamber matching the same set of operational states and transitions upon which the model was based. The prototype was initially heated under no load to $100^{\circ} \mathrm{C}$ at which time the length of the prototype in the austenite free state $\mathrm{L}_{\mathrm{A} 0}$ (figure 2(a)) was measured between the steel ring attachments using a US digital linear encoder strip with 250 divisions per inch. The width of the entire prototype $W_{\text {totalA0 }}$ was measured using digital calipers. A weight was attached to the prototype around a pulley, resulting in extension of the austenitic prototype into the austenite extended state (figure 2(b)) and the length $L_{\text {Aext }}$ was recorded. The environmental chamber and the prototype were cooled to $20^{\circ} \mathrm{C}$, the prototype continued to extend into the martensite extended state (figure 2(c)), and length $L_{\text {Mext }}$ was recorded. Heating the environmental chamber and prototype to $100^{\circ} \mathrm{C}$ caused the prototype to contract under load into the austenite contracted state (figure 2(d)), with a measured length of $L_{\text {Acont }}$. The weight was removed and the prototype returned to the austenite free state at which time a larger load was
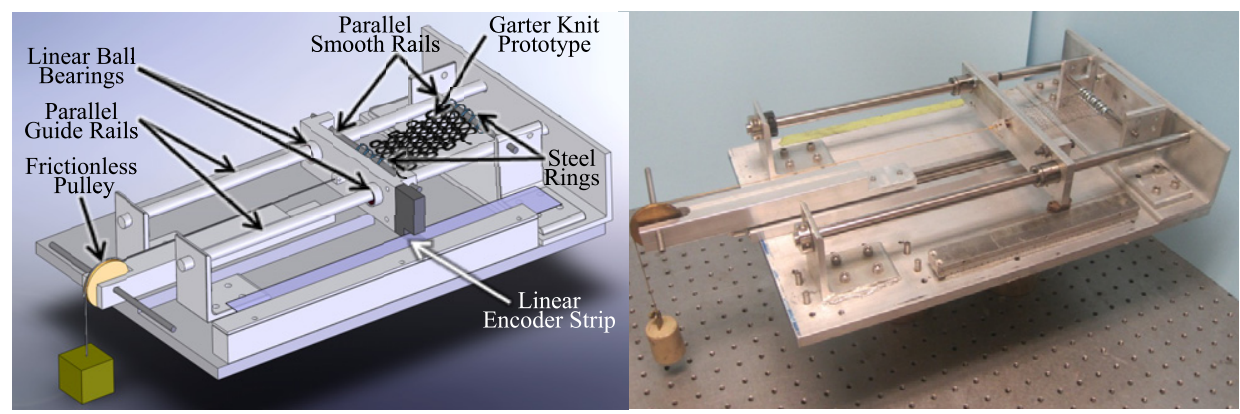

Figure 8. Experimental setup (schematic on left, experimental on right) used to determine the load-extension behavior of the garter stitch states and the resulting actuation behavior. 


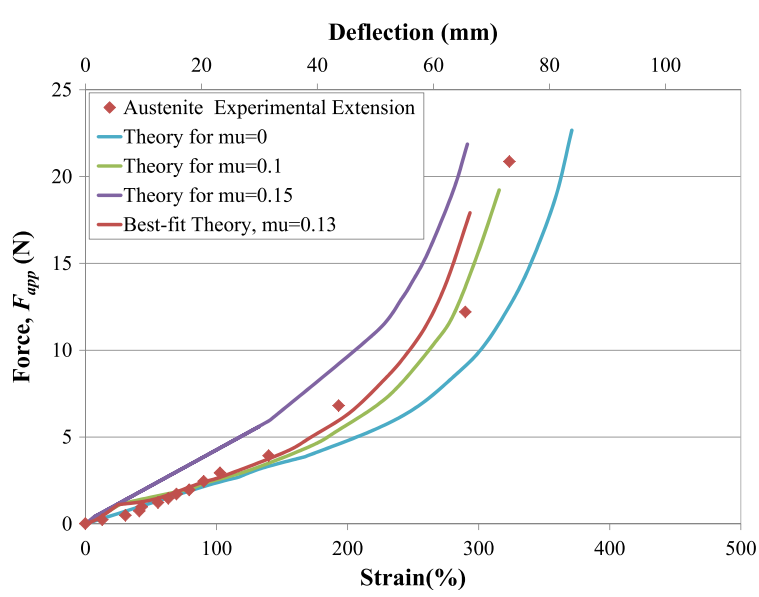

Figure 9. Austenite extended state. Theoretical and experimental results agree with an average relative displacement error of $6.1 \%$ with coefficient of friction $\mu=0.13$.

applied to the prototype and the testing process was repeated. This sequence ensures that the friction is acting against the elongation of the knit during extension and against contraction during actuation.

\subsection{Austenite and martensite extended states}

The experimental and theoretical results are shown in figure 9 for austenite extended states and figure 10 for the martensite extended states. Both the theory and experimental results of the austenite and martensite extended states displayed ' $J$ ' shaped load-extension curves similar to the load-extension curves of passive knits [74, 75]. For each experimentally applied load $\left(F_{\text {app }}\right)$ the knit prototype underwent a deflection from the austenite free state, $\Delta_{\text {Aext }}=L_{\mathrm{Aext}}-L_{\mathrm{A} 0}$ for the austenite extended state deflection and $\Delta_{\mathrm{Mext}}=L_{\mathrm{Mext}}-L_{\mathrm{A} 0}$ for the martensite extended state deflection. During lower relative force loadings the knit underwent extreme stretching, up to approximately $200 \%$ strain for a $6 \mathrm{~N}$ applied load for the austenite extended state and $250 \%$ strain for a $3 \mathrm{~N}$ applied load for the martensite extended state. As the applied load was further increased the knit stiffened, the loops continued to stretch (up to $300 \%$ strain for the austenite extended state and $330 \%$ strain for the martensite extended state under an applied load of $15 \mathrm{~N}$ ), but at a decreased rate because the loops experienced less change in curvature and more longitudinal alignment of the legs of the loop.

Using a friction value of $\mu=0.13$, both the austenite and martensite theoretical load-extension curves provided excellent correlation with the experimental data with an average relative displacement error of $6.1 \%$ and an average absolute displacement error of $1.9 \mathrm{~mm}$ over the experimental range of applied loads $(0-20 \mathrm{~N})$ for the austenite extended state and $4.1 \%$ average relative displacement error and $1.8 \mathrm{~mm}$ average absolute displacement error over the experimental range of applied loads $(0-12 \mathrm{~N})$ for the martensite extended state. The coefficient of friction $(\mu=0.13)$ was found independently for both the austenite extended state and the martensite extended state using a least squares analysis best

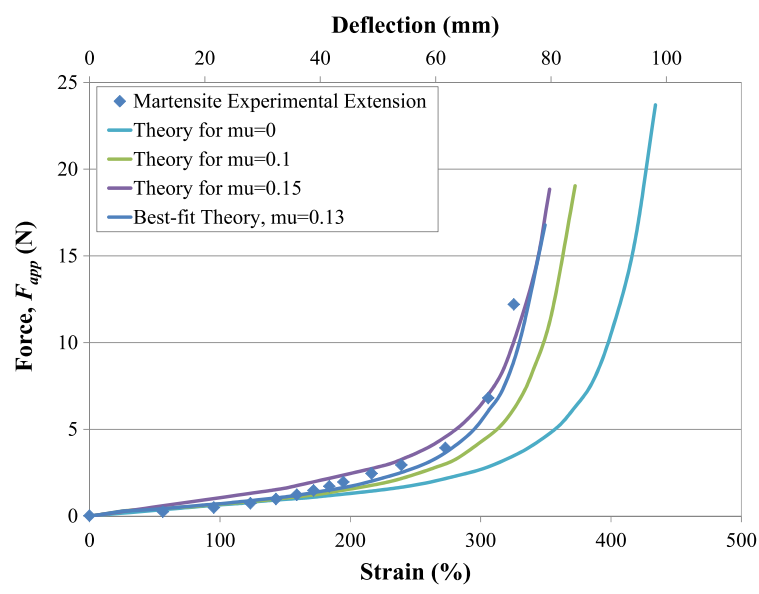

Figure 10. Martensite extended state. Theoretical and experimental results agree with an average relative displacement error of $4.1 \%$ with coefficient of friction $\mu=0.13$.

fit over a range of coefficients between $\mu=0.1$ and 0.15 . The bounding coefficients of friction were chosen based on published values for the friction of smooth stainless steel materials $[85,86]$. A range of friction values were investigated because it is difficult to accurately measure the coefficient of friction between two wires. A coefficient of friction of $\mu=$ 0.13 was independently found to be the best representation of the friction in each of the austenite and martensite extended states, supporting the physical correctness of this parameter as opposed to a pure fit parameter. The theoretical load-extension results for the two friction cases bounded the majority of the experimental data points in both the austenite and martensite extended states. The smaller friction value, $\mu=0.1$, resulted in an average relative displacement errors of $10.4 \%$ for austenite and $8.6 \%$ for martensite over the range of applied loads for each state, while the larger friction value, $\mu=0.15$, resulted in average relative displacement errors of $18.6 \%$ for austenite and $7.5 \%$ for martensite. All theoretical calculations involving friction provided vast improvements over the frictionless theory which was considerably less stiff than the experiments with an average relative displacement error of $22.3 \%$ for the austenite extended state and $22.5 \%$ for the martensite extended state over the range of applied loads for each state. While previous research has shown strong correlations between experimental and theoretical frictionless results (within 10\% average displacement error $[74,75]$ ), the inclusion of friction in the modeling of the load-deflection of active knits vastly improved the average displacement error, reducing the error by 3.7 times for the austenite extended state and 5.5 times for the martensite extended case. Slippage between loops was observed during the operational transitions into the austenite and martensite extended states, validating the friction assumptions used in the model.

\subsection{Austenite contracted state}

The operational transition into the austenite contracted state from the martensite extended state included sticking of the interlacing contact point. The sticking of adjacent loops 


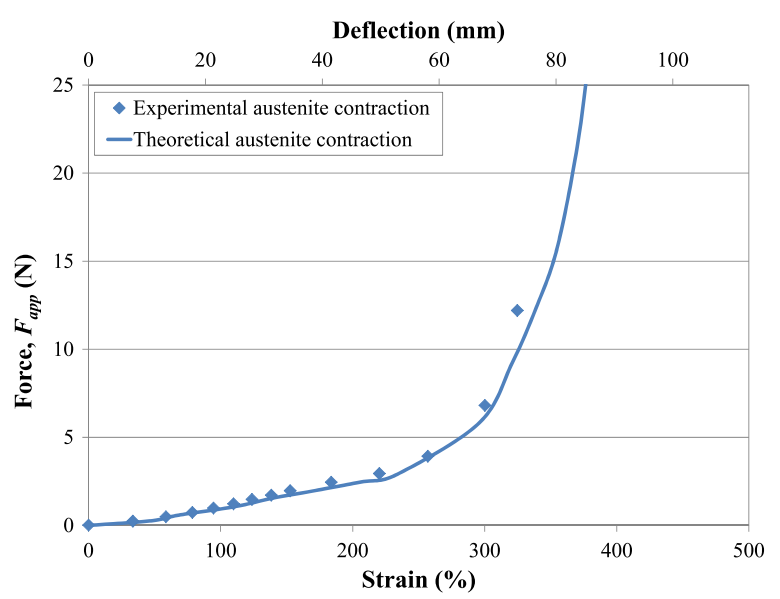

Figure 11. Austenite contracted state. Theoretical and experimental results agree with an average relative displacement error of $2.8 \%$.

prohibited loops from sliding past one another, thus the actuation motion was solely a result of the loops straightening. The theoretical austenite contracted states were assembled to produce a load-extension relationship where the load is the applied force the knit actuates against $\left(F_{\text {app }}\right)$ and the deflection $\left(\Delta_{\text {Acont }}=L_{\text {Acont }}-L_{\mathrm{A} 0}\right)$ is the difference between the recovered length and the length of the austenite free state. This theoretical load-extension relationship produces another ' $\mathrm{J}$ ' shaped curve which matches the experimental results in form and magnitude (figure 11). Both the theoretical and experimental austenite contracted results are stiffer than that of the martensite extended load-extension curve, but less stiff than the austenite extended curve: the austenite contracted knit stretched under low force loadings to $220 \%$ strain for a $3 \mathrm{~N}$ applied load before becoming stiffer as the knit stretched to $320 \%$ strain under a $15 \mathrm{~N}$ applied load. The loops were observed to stick during the operational transition into the austenite contracted state, validating the friction assumptions used in the model for this state. The theoretical austenite contracted curve quantitatively matches the experimental extremely closely with an average relative displacement error of $2.8 \%$ with an average absolute displacement error of $1.8 \mathrm{~mm}$ over the range of applied forces actuated against, $0-12 \mathrm{~N}$.

\subsection{Net actuation}

The net actuation performance $\left(\Delta_{\text {act }}=\Delta_{\text {Mext }}-\Delta_{\text {Acont }}\right)$ is obtained by subtracting the displacement of the austenite contracted state from the martensite extended state at each applied load, as this is the motion obtained when actuating under a constant load. The theoretical and experimental actuation curves show the same distinctive qualitative performance (figure 12). As the load increased the net actuation strain increased to a maximum peak actuator strain then decreased under increased applied loads as the prototype was not able to recover as much of the martensite strain. The shape of the actuation curves is unique: for small deflections, where the loops do not change shape much, net displacement increases as load increases since

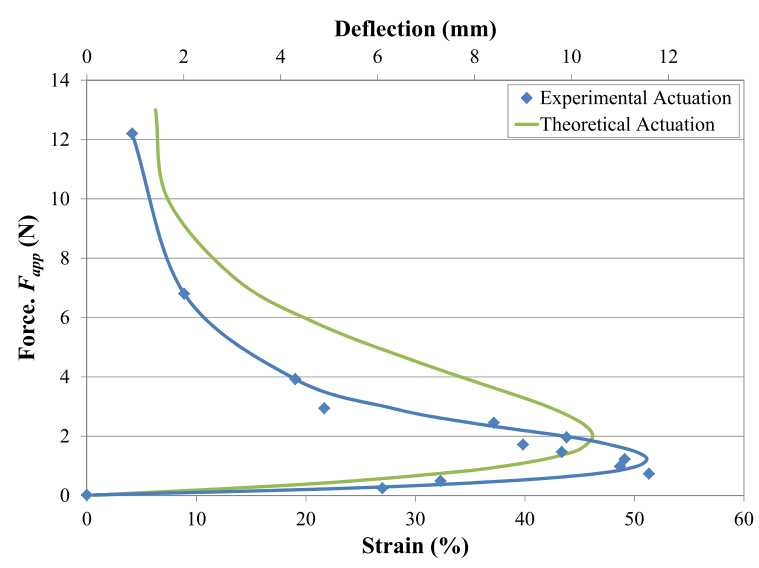

Figure 12. Garter knit actuation results. Knit prototype transitions between martensite extended and austenite contracted states to create theoretical actuation curve with same form as experimental results with an average absolute displacement error of $2.0 \mathrm{~mm}$.

the performance is dominated primarily by the difference in stiffness between the two material phases. Whereas for larger deflections, where the loops are narrow and elongated, larger loads produce smaller net deflections since the austenite phase loses its ability to recover motion through the reduced leverage of the elongated loops. This implies that there is an optimal deflection point for designing such actuators to provide the maximum possible deflection (and a similar, but differently located configuration for maximum work) for which the predictive model can aid in designing the ideal architecture for a given application.

The theoretical and experimental actuation curves are also an excellent quantitative match given the complex behavioral form and assumptions made during the development of the analytical model. The peak actuator strain, $51.2 \%$, was observed under a $1.22 \mathrm{~N}$ applied load while, theoretically, a $46.2 \%$ peak actuator strain was predicted under a $2 \mathrm{~N}$ applied load. The model accurately predicts the peak actuator motion within $5 \%$ strain at a higher applied force than experimentally witnessed. The experimental and theoretical actuation curves correlate with an average absolute displacement error of $2.0 \mathrm{~mm}$ over the $12 \mathrm{~N}$ range of applied actuation forces which is slightly larger compared to $1.8 \mathrm{~mm}$ over each of the martensite extended and austenite contracted states. The differences between the theoretical and experimental actuation results stem from compounding the displacement errors for both the martensite extended and austenite contracted states through subtraction during the calculation of the actuation behavior and also result from the assumptions made during modeling, particularly, state dependent stick-slip friction, full material transition into each state, and linear elastic material behavior. The friction assumption of constant sliding during extension gives larger strains than experimentally witnessed while the constant sticking assumption allows more force to be transferred under restricted motions consistent with the validation shown in figure 12. The validity of the small strain assumption (and therefore the homogeneous phase and linear elastic assumptions) can be estimated from the loop curvature by 
examining the ratio of the wire diameter, $d$, to the loop diameter as approximated by the knitting needle diameter, $D$. For the validation prototype, this ratio is 0.037 , indicating that the largest strains, along the outer surface of the bent wire, violate the small strain assumption. However, in the austenite extended state the loops straighten significantly, reducing the maximum strain, and most of the material, even in the martensite extended state, will experience lower strains. Even with these assumptions the prediction was quite good, capturing the kinematic loop shape change and the kinetic performance of the contractile active knit.

Large actuation motions with unprecedented strains were observed during all the experiments. The knit architecture significantly amplified the strain of a bulk individual fiber (in the case of SMA $8 \%$ recoverable and 3-4\% actuation), to as much as $250 \%$ recoverable strain allowed for moderate forces of tens of Newtons and $51 \%$ actuation strain (figure 12) under a $1.2 \mathrm{~N}$ applied load. The knitted actuator also provides enhanced force performance, axially contracting SMA wires 8 mil in diameter operate at a maximum strain of $4 \%$ under a $5.8 \mathrm{~N}$ applied load while the active knit prototype generated $4.1 \%$ strain under a large applied load of $12.2 \mathrm{~N}$. The active knit actuator enhanced the strain by an order of magnitude and the force by two times that of the same diameter straight SMA wires, affording enhanced actuation capabilities to a variety of application domains and enabling new technologies.

\section{Conclusions}

This paper presents a state-based model that can determine the actuation behavior of garter stitch active knitted actuators fabricated from variable stiffness smart material wire. The model determines the states experienced during a typical actuation cycle: austenite free, austenite extended, martensite extended, and martensite contracted states while considering the mechanical and thermal operational transitions the textile undergoes during deformation. The model assumes different friction conditions (stick or slip) during the operational transition depending on the loading and the initial state of the textile. Elastica theory and Euler-Bernoulli beam bending are used in addition to the knit geometry, force-equilibrium and the governing differential equations to determine the deflection from the austenite free state of the textile and thus the relative displacement between the martensite extended and austenite contracted states for actuation. A set of fully analytical transcendental algebraic equations (as opposed to a set of coupled differential equations) describe the deflections experienced within a unit cell for each operational state, and are scaled to produce the load-extension properties of the entire textile for each state as well as the net actuation performance, providing a model that can be used for design and optimization of active garter stitch knits.

A prototype knit textile was fabricated from $70^{\circ} \mathrm{C}$ Flexinol ${ }^{\circledR}$ wire and used to validate the model in an experimental study where the textile was cycled through the modeled sequence of operational states under a variety of external loads from 0 to $12 \mathrm{~N}$. The model provided an excellent match to the experimental results particularly considering the modeling assumptions: dual stiffness, linear elastic material with prescribed friction states. The martensite and austenite extended states were predicted with an average relative displacement error of $4.1 \%$ and $6.1 \%$ using a coefficient of friction $\mu=0.13$ as found independently via a best fit analysis for each the martensite and austenite extended states. The theoretical austenite contracted state agreed with the experimental results with a $2.8 \%$ average relative displacement error. The results validate the observed friction requirements-sliding for extended states and sticking for contracted states. The theoretical actuation, the difference between the martensite extended and austenite contracted states, was found to match the form and magnitude of the experimental results with an average absolute displacement error of $2.0 \mathrm{~mm}$.

The analytical model presented in this paper provides a tractable tool for the prediction, design, and tailoring of active garter stitch knitted actuators. The unique shape of the actuation curve with increasing net displacement versus force at low load, a maximum displacement peak, and decreasing displacement at further increasing loads provides the opportunity for tailoring of architectural parameters such as loop size, wire diameter, and the number of courses and wales within the textile to optimally match the specific needs of a particular application. This highly leveraged architecture allows for large strains $(51 \%)$ at moderate forces $(1.22 \mathrm{~N})$ and usable strains $(4.1 \%)$ at enhanced forces $(12 \mathrm{~N})$ over the material alone (4\% strain at $5.8 \mathrm{~N}$ ) [87]. This new actuation capability goes beyond what is possible with conventional actuation technology and the current state of the art in smart materials actuators, meeting increasing needs for large contractile actuation surfaces.

\section{Acknowledgments}

The authors would like to acknowledge the National Science Foundation for fellowship support of the lead author and the Air Force Office of Scientific Research for their support under grant FA9550-09-1-0217.

\section{References}

[1] Gandhi F and Anusonti-Inthra P 2008 Skin design studies for variable camber morphing airfoils Smart Mater. Struct. 17015025

[2] McKnight G, Doty R, Keefe A, Herrera G and Henry C 2010 Segmented reinforcement variable stiffness materials for reconfigurable surfaces J. Intell. Mater. Syst. Struct. 21 1783-93

[3] Rodriguez A R 2007 Morphing aircraft technology survey AIAA Aerospace Sciences Mtg and Exhibit (Reno, NV) pp 1-16

[4] NASA 2011 NASA Fact Sheet: Opening the Door to a New Era in Flight, FS-2001-04-63-LaRC (Hampton, VA: NASA Langley Research Center)

[5] Morgan N B 2004 Medical shape memory alloy applications-the market and its products Mater. Sci. Eng. A 378 16-23

[6] Hoh D J, Hoh B L, Amar A P and Wang M Y 2009 Shape memory alloys: metallurgy, biocompatibility, and 
biomechanics for neurosurgical applications Neurosurgery 64 199-215

[7] Veeramani A S, Buckner G D, Owen S B, Cook R C and Bolotin G 2008 Modeling the dynamic behavior of a shape memory alloy actuated catheter Smart Mater. Struct. 17015037

[8] Todoroki A, Kumagai K and Matsuzaki R 2009 Self-deployable space structure using partially flexible CFRP with SMA wires J. Intell. Mater. Syst. Struct. 20 1415-24

[9] Ganesh Raja M and Narayanan S 2009 Simultaneous optimization of structure and control of smart tensegrity structures J. Intell. Mater. Syst. Struct. 20 109-17

[10] Sofla A Y N, Elzey D M and Wadley H N G 2007 A rotational joint for shape morphing space truss structures Smart Mater. Struct. 16 1277-84

[11] Yoo E, Roh J and Han J 2007 Wrinkling control of inflatable booms using shape memory alloy wires Smart Mater. Struct. $16340-8$

[12] Yoon H and Washington G 2010 An optimal method of shape control for deformable structures with an application to a mechanically reconfigurable reflector antenna Smart Mater. Struct. 19105004

[13] Huber J, Fleck N and Ashby M 1997 The selection of mechanical actuators based on performance indices Proc. R. Soc. A 453 2185-205

[14] Wada T, Lee C C, Chen H H, Kusaka M and Taya M 2003 Design of spring actuators made of ferromagnetic shape memory alloys and composites Proc. SPIE 5054 125-34

[15] Sutou Y, Oikawa K, Kainuma R, Ishida K and Taya M 2003 Martensitic transformation behavior under magnetic field of Co-Ni-Al ferromagnetic shape memory alloys Proc. SPIE $\mathbf{5 0 5 3} 159-68$

[16] Liang Y, Taya M and Kuga Y 2003 Design of diaphragm actuator based on ferromagnetic shape memory alloy composite Proc. SPIE 45 45-52

[17] Taya M, Wada T and Lee C C 2004 Design of torque actuators based on ferromagnetic shape memory alloy composites Proc. SPIE 5390 309-16

[18] Spinks G M, Wallace G G, Ding J, Dezhi Z, Xia B, Scott T and Truong V 2003 Electroactive polymer actuator devices (EAPAD) Proc. SPIE $505121-8$

[19] Metz P, Alici G and Spinks G 2006 A finite element model for bending behaviour of conducting polymer electromechanical actuators Sensors Actuators A 130/131 1-11

[20] Alici G and Huynh N M 2006 Predicting force output of trilayer polymer actuators Sensors Actuators A 132 616-25

[21] Fernandez D, Moreno L and Baselga J 2005 A bioinspired EAP actuator design methodology Proc. SPIE 5759 361-70

[22] Bar-Cohen Y, Sherritl S and Lih S 2001 Characterization of the electromechanical properties of EAP materials Proc. SPIE 4329 319-27

[23] Bar-Cohen Y 2002 Electro-active polymers: current capabilities and challenges Proc. SPIE 4695 1-7

[24] Haertling G H 1994 Rainbow ceramics-a new type of ultrahigh-displacement actuator Am. Ceram. Soc. Bull. 73 93-6

[25] Barron B W, Li G and Haertling G H 1996 Temperature dependent characteristics of cerambow actuators Proc. 10th IEEE Int. Symp. Applications of Ferroelectrics vol 1, pp 305-8

[26] Hall S R and Prechtl E F 1996 Development of a piezoelectric servo-flap for helicopter rotor control Smart Mater. Struct. 5 26-34

[27] Chandran S, Kugel V D and Cross L E 1997 CRESCENT: a novel piezoelectric bending actuator Proc. SPIE 3041 461-9
[28] Kugel V D, Chandran S and Cross L E 1997 Comparative analysis of piezoelectric bending-mode actuators Proc. SPIE 3040 70-80

[29] Ervin J D and Brei D 1998 Recurve piezoelectric strain-amplifying actuator architecture IEEE/ASME Trans. Mechatronics 3 293-301

[30] Moskalik A J and Brei D 1998 Parametric investigation of the deflection performance of serial piezoelectric cblock actuators J. Intell. Mater. Syst. Struct. 9 223-31

[31] Vendlinski J and Brei D 2003 Dynamic behavior of telescopic actuators J. Intell. Mater. Syst. Struct. 14 577-85

[32] Bamford R, Kuo C P, Glaser R and Wada B K 1995 Long stroke precision PZT actuator AIAA/ASME/ASCE/AHS/ASC Structures, Structural Dynamics, and Materials Conf. pp 3278-84

[33] Onitsuka K, Dogan A, Tressler J F, Xu Q, Yoshikawa S and Newnham R E 1995 Metal-ceramic composite transducer, the 'moonie' J. Intell. Mater. Syst. Struct. 6 447-55

[34] Dogan A, Fernandez J F, Uchino K and Newnham R E 1996 Cymbal' electromechanical actuator Proc. IEEE Int. Symp. on Applications of Ferroelectrics vol 1, pp 213-6

[35] Prechtl E F and Hall S R 1999 Design of a high efficiency, large stroke, electromechanical actuator Smart Mater. Struct. 8 13-30

[36] Hall S R, Tzianetopoulou T, Straub F and Ngo H 2000 Design and testing of a double X-frame piezoelectric actuator Proc. SPIE 3985 26-37

[37] Park J, Carman G P and Thomas Hahn H 2000 Design and testing of a mesoscale piezoelectric inchworm actuator with microridges J. Intell. Mater. Syst. Struct. 11 671-84

[38] Lobontiu N, Goldfarb M and Garcia E 2001 Piezoelectric-driven inchworm locomotion device Mech. Mach. Theory 36 425-43

[39] Hartmut J 1997 Adaptronics and Smart Structures: Basics, Materials, Design, and Applications (Berlin: Springer)

[40] Tohyama O, Maeda S, Abe K and Murayama M 2001 Shape memory alloy actuators and their reliability Proc. SPIE $4592111-8$

[41] Li L and Sottos N R 1994 Consideration of electromechanical coupling in the prediction of 1-3 piezocomposite properties Proc. ASME IMECE 193 23-32

[42] Bent A A, Hagood N W and Rodgers J P 1995 Anisotropic actuation with piezoelectric fiber composites J. Intell. Mater. Syst. Struct. 6 338-49

[43] Maclean B J and Jacobsen S C 1995 Active fibers for development of adaptive structures ARPA Actuator Issues Workshop

[44] Fernandez J F, Dogan A, Zhang Q M, Tressler J F and Newnham R E 1995 Hollow piezoelectric composites Sensors Actuators A 51 183-92

[45] Zhang Q, Wang H and Cross L E 1993 Piezoelectric tubes and tubular composites for actuator and sensor applications J. Mater. Sci. 28 3962-8

[46] Herold-Schmidt U, Floeth E, Last B, Schaefer W and Zaglauer H W 1997 Qualification of smart composites for use in aerospace applications Proc. SPIE 3044 168-75

[47] Jang B Z 1993 Intelligent composites and structures-a review Proc. Int. Conf. Advanced Composite Materials pp 661-7

[48] Chen P C and Chopra I 1996 Induced strain actuation of composite beams and rotor blades with embedded piezoceramic elements Smart Mater. Struct. 5 35-48

[49] Agrawal S K, Tong D and Nagaraja K 1994 Modeling and shape control of piezoelectric actuator embedded elastic plates J. Intell. Mater. Syst. Struct. 5 514-21

[50] Ray M C and Reddy J N 2005 Active control of laminated cylindrical shells using piezoelectric fiber reinforced composites Compos. Sci. Technol. 65 1226-36

[51] Brei D and Cannon B, Piezoceramic hollow fiber active composites Compos. Sci. Technol. 64 245-61 
[52] Cannon B and Brei D 2000 Feasibility study of microfabrication by coextrusion (MFCX) hollow fibers for active composites J. Intell. Mater. Syst. Struct. 11 659-70

[53] Cannon B and Brei D 2002 Piezoceramic hollow fiber active composites AIAA/ASME/ASCE/AHS/ASC Structures, Structural Dynamics, and Materials Conf. pp 1-18

[54] Schetky L McD and Wu M H, Properties and processing of shape memory alloys for use as actuators in intelligent composite materials Smart Struct. Mater. Am. Soc. Mech. Eng. vol 24 65-71

[55] Baz A, Chen T and Ro J 1994 Shape control of nitinol-reinforced composite beams Proc. SPIE 2190 436-53

[56] Baz A and Chen T 2003 Active control of the lateral buckling of Nitinol-reinforced composite beams Proc. SPIE $242730-48$

[57] Jonnalagadda K D, Sottos N R, Qidwai M A and Lagoudas D C 1998 Transformation of embedded shape memory alloy ribbons J. Intell. Mater. Syst. Struct. 9 1-23

[58] Sottos N R, Kline C E, Qidwai M A and Lagoudas D C 1996 Analysis of phase transformation fronts in SMA composites Proc. SPIE 2715 427-38

[59] Jonnalagadda K and Sottos N R 2003 Influence of adhesion on micromechanical behavior of SMA composites Proc. SPIE 2442 143-51

[60] Boussu F, Bailleul G, Petitniot J and Vinchon H 2002 Development of shape memory alloy fabrics for composite structures AUTEX Technical Textiles Int. Conf. vol 51, pp 1-7

[61] Evans J, Brei D and Luntz J 2006 Preliminary experimental study of SMA knitted actuation architectures Proc. ASME IMECE 15409 369-76

[62] Postle R and Munden D L 1967 Analysis of the dry-relaxed knitted loop configuration-part II: three-dimensional analysis J. Text. Inst. 58 352-65

[63] Postle R and Munden D L 1967 Analysis of the dry relaxed knitted loop configuration-part I: two-dimensional analysis J. Text. Inst. 58 329-51

[64] Leaf G A V 1960 Models of the plain-knitted loop J. Text. Inst. 51 T49-58

[65] Leaf G A V and Glaskin A 1953 The geometry of a plain knitted loop J. Text. Inst. 44 T587-605

[66] Peirce F T 1947 Geometrical principles applicable to the design of functional fabrics J. Text. Inst. 17 123-47

[67] Hearle J W S, Grosberg P and Backer S 1969 Structural Mechanics of Fibers, Yarns, and Fabrics (New York: Wiley Interscience) p 80

[68] Peirce F T 1937 The geometry of cloth structure J. Text. Inst. 28 T45-96

[69] Munden D L 1959 The geometry and dimensional properties of plain-knit fabrics J. Text. Inst. 50 T448-71

[70] Smirfitt J A 1965 Worsted $1 \times 1$ rib fabrics-part I: dimensional properties J. Text. Inst. 56 T248-59
[71] de Jong S and Postle R 1977 Energy analysis of knitted-fabric mechanics by means of optimal control theory-part I: the nature of loop-interlocking in the plain-knitted structure J. Text. Inst. 68 307-15

[72] de Jong S and Postle R 1977 Energy analysis of knitted-fabric mechanics by means of optimal control theory-part II: relaxed-fabric dimensions and tensile properties of the plain-knitted structure J. Text. Inst. 68 316-23

[73] de Jong S and Postle R 1977 Energy analysis of knitted-fabric mechanics by means of optimal control theory-part III: the $1 \times 1$ rib-knitted structure J. Text. Inst. 68 324-9

[74] Shanahan W J and Postle R 1974 A theoretical analysis of the tensile properties of plain-knitted fabrics - part I: the load-extension curve for fabric extension parallel to the courses J. Text. Inst. 65 200-12

[75] Hong H, de Araujo M D, Rangueiro R and Ciobanu O 2002 Theoretical analysis of load-extension properties of plain weft knits made from high performance yarns for composite reinforcement Textile Res. J. 72 991-5

[76] Padaki N V, Alagirusamy R and Sugun B S 2006 Knitted preforms for composite applications J. Indust. Text. 35 295-321

[77] Love A 1927 A Treatise on the Mathematical Theory of Elasticity (Cambridge: Cambridge University Press)

[78] Ramakrishna S 1997 Characterization and modeling of the tensile properties of plain weft-knit fabric reinforced composites Compos. Sci. Technol. 57 1-22

[79] Leong K H, Ramakrishna S, Huang Z M and Bibo G A 2000 The potential of knitting for engineering composites-a review Composites A 31 197-220

[80] Wu W L, Hamada H, Kotaki M and Maekawa Z 1995 Design of knitted fabric reinforced composites J. ReinfPlast. Comp 14 786-98

[81] Huang Z M, Ramakrishna S and Leong K H 2002 Modeling the tensile behavior of milano rib knit fabric composites J. Reinforced Plast. Compos. 21 1123-46

[82] Luo Y and Verpoest I 2002 Biaxial tension and ultimate deformation of knitted fabric reinforcements Composites A 33 197-203

[83] Dynalloyinc, Specs on Various sizes of Flexinol www. dynalloy.com/pdfs/MWPBv4.00_FlexSpecs.pdf accessed 26 May 2009

[84] Shaw J 2002 A thermomechanical model for a 1-D shape memory alloy wire with propagating instabilities Int. J. Solids Struct. 39 1275-305

[85] Weber R L, Manning K V and White M W 1965 College Physics 4th edn (New York: McGraw-Hill) p 66

[86] 2006 Engineer's Handbook, Coefficient of Friction Reference Table www.engineershandbook.com/Tables/ frictioncoefficients.htm accessed 24 May 2006

[87] 2009 Dynalloy, Technical Characteristics of Flexinol Actuator Wires www.dynalloy.com/pdfs/TCF1140RevD.pdf accessed 21 June 2009 